\title{
Dynamic trading under integer constraints
}

\author{
Stefan Gerhold ${ }^{1}$ Paul Krühner ${ }^{2}$
}

Received: 4 September 2017 / Accepted: 18 April 2018 / Published online: 21 August 2018

(C) The Author(s) 2018

\begin{abstract}
In this paper, we investigate discrete-time trading under integer constraints, that is, we assume that the offered goods or shares are traded in integer quantities instead of the usual real quantity assumption. For finite probability spaces and rational asset prices, this has little effect on the core of the theory of no-arbitrage pricing. For price processes not restricted to the rational numbers, a novel theory of integerarbitrage-free pricing and hedging emerges. We establish an FTAP, involving a set of absolutely continuous martingale measures satisfying an additional property. The set of prices of a contingent claim is not necessarily an interval, but is either empty or dense in an interval. We also discuss superhedging with integer-valued portfolios.
\end{abstract}

Keywords Arbitrage $\cdot$ Hedging $\cdot$ Integer constraints

Mathematics Subject Classification (2010) 91G10 - 91G20 $11 \mathrm{~K} 60$

JEL Classification $\mathrm{G} 12 \cdot \mathrm{C} 02 \cdot \mathrm{C} 65$

\section{Introduction}

Classical, frictionless no-arbitrage theory $[9,16]$ makes several simplifying assumptions on financial markets. In particular, position sizes may be arbitrary real numbers,

S. Gerhold gratefully acknowledges financial support from the Austrian Science Fund (FWF) under grants P 24880 and P 30750.

\begin{tabular}{ll}
\hline & P. Krühner \\
& p.eisenberg@liverpool.ac.uk \\
& S. Gerhold \\
& sgerhold@ fam.tuwien.ac.at \\
1 & TU Wien, Vienna, Austria \\
2 & University of Liverpool, Liverpool, UK
\end{tabular}


which allows trading strategies that cannot be implemented in practice. Even if brokers are receptive to fractional amounts of shares, there will be a smallest fraction that can be purchased or sold. Moreover, traders might wish to avoid odd lots because of additional brokerage fees and the usually poor liquidity of small positions. In this case, the smallest traded unit would be a round lot consisting of several (e.g. 100) shares. Both situations can be covered by assuming that integer amounts of a price process $\left(S_{t}^{1}, \ldots, S_{t}^{d}\right)_{t \in \mathbb{T}}$ can be traded. The set of trading times $\mathbb{T}$ is assumed to be finite in this paper. For simplicity, we call $S^{i}$ the price process of the $i$ th (risky) asset, although it may have the interpretation of a fraction or a round lot of an actual asset price. We assume that the amount of money in the riskless asset may take arbitrary real values. On the one hand, this increases tractability; on the other hand, it makes economic sense as the smallest possible modification of the bank account is usually several orders of magnitude smaller than that of the risky positions. Thus our integer trading strategies in a model with $d$ risky assets take values in $\mathbb{R} \times \mathbb{Z}^{d}$ at each time. For some results and proofs, we also consider rational strategies with values in $\mathbb{R} \times \mathbb{Q}^{d}$. By clearing denominators, the corresponding notions of freeness of arbitrage are equivalent (see Lemma 2.5).

The bulk of the existing literature on arbitrage, pricing and hedging under trading constraints (see e.g. [8, 12], [13, Chap. 9]) imposes convexity assumptions on the set of admissible strategies, which are unrelated to integer constraints. An exception is the paper by Bender and Kohlmann [5], who study superhedging under constraints in a multi-dimensional diffusion model. They mention explicitly that their assumptions cover integer strategies [5, Example 4.4], and show that any contingent claim that admits a constrained superhedge has an optimal constrained superhedge [5, Theorem 3.1]. We shall see below (Example 5.3) that there are discrete-time models that do not have this property. We also mention the paper by Deng et al. [11], who show that deciding the existence of arbitrage in a one-period model under integer constraints is an NP-hard problem.

Integer constraints feature prominently in the computational finance literature, e.g. in the papers $[3,6,7]$ which employ mixed-integer nonlinear programming to solve the Markowitz portfolio selection problem. In the literature, other keywords such as minimum lot restrictions, minimum transaction level and integral transaction units are used with the same meaning as our integer constraints.

In our main results, we assume that the underlying probability space is finite (Assumption 2.1 of Sect. 2). This assumption is realistic, because actual asset prices move by ticks, and prices larger than $10^{10^{10}}$, say, will never occur. Still, extending the material to arbitrary probability spaces might be mathematically interesting, but is left for future work.

In Sect. 2, we introduce the notions of no integer arbitrage (NIA) and no integer free lunch (NIFL) in a straightforward way. It turns out (Theorem 2.7) that the latter property is equivalent to the classical no-arbitrage condition NA, and so we concentrate on NIA in the rest of the paper. Our first main result is a fundamental theorem of asset pricing (FTAP; Theorem 2.13) characterising NIA. It involves a set of absolutely continuous martingale measures satisfying an additional property. If the martingale measures are chosen with maximal support, then the additional property is that there is no integer arbitrage (but possibly a real arbitrage) outside the support of the martingale measure. Moreover, there is no real arbitrage on the (never empty) support of 
the martingale measure. The theorem is thus not as neat as the classical FTAP, but is still useful for establishing several of our subsequent results. In Sect. 3, we define the set $\Pi_{\mathbb{Z}}(C)$ of NIA-compatible prices of a claim $C$. The integer variant of the classical representation using the set of equivalent martingale measures features only an inclusion instead of an equality (Proposition 3.4), and in fact $\Pi_{\mathbb{Z}}(C)$ may be empty. Even if it is nonempty, it need not be an interval; however, $\Pi_{\mathbb{Z}}(C)$ is then always dense in an (explicit) interval, which is the main result of Sect. 4. As regards methodology, many of our arguments just use the countability of $\mathbb{Z}^{d}$ (and $\mathbb{Q}^{d}$ ), or the denseness of $\mathbb{Q}^{d}$ in $\mathbb{R}^{d}$. Still, at some places (such as Lemma 2.6, Example 5.3 and Theorem 5.4), we invoke non-trivial results from number theory, collected in Appendix.

Readers who are mainly interested in the practical consequences of integer restrictions are invited to read (besides the basic definitions) Theorem 2.8, Theorem 3.6 and Sect. 5. In a nutshell, for the discrete-time models used in practice (finite probability space, floating-point-i.e., rational-asset values), the core of no-arbitrage theory does not change much. One exception is the fact that the supremum of claim prices consistent with no-integer-arbitrage need not agree with the smallest integer superhedging price (see Sect. 5). Still, this property holds in a limiting sense when superhedging a large portfolio of identical claims. That said, our work is by no means the last word on the practical consequences of integer restrictions in dynamic trading. Problems such as quantile hedging, hedging with risk measures or hedging under convex constraints may well be worth studying under integer restrictions. In Sect. 6, we discuss a toy example of variance-optimal hedging under integer constraints, which leads to the closest vector problem (CVP), a well-known algorithmic lattice problem.

\section{Trading strategies and absence of arbitrage}

We work with a probability space $(\Omega, \mathcal{A}, P)$. Our main results use the following standing assumption:

Assumption 2.1 $\Omega$ is finite, $\mathcal{A}$ is the power set of $\Omega, P[\{\omega\}]>0$ for any $\omega \in \Omega$, and we choose an enumeration $\omega_{1}, \ldots, \omega_{n}$ of its elements.

We assume that there is a finite set of times $\mathbb{T}:=\{0, \ldots, T\}$, with $T \in \mathbb{N}$, at which trading may occur, and fix a filtration $\left(\mathcal{F}_{t}\right)_{t \in \mathbb{T}}$, where $\mathcal{F}_{T}=\mathcal{A}$ and $\mathcal{F}_{0}=\{\emptyset, \Omega\}$. The (deterministic) riskless interest rate is $r>-1$, and we have $d$ risky assets with prices $S_{t}=\left(S_{t}^{1}, \ldots, S_{t}^{d}\right)$ at time $t \in \mathbb{T}$, where $S_{t}$ is assumed to be nonnegative and $\mathcal{F}_{t}$-measurable. The price of the riskless asset is denoted by $S_{t}^{0}:=(1+r)^{t}$ for $t \in \mathbb{T}$, and we denote the market price processes by $\bar{S}:=\left(S^{0}, S\right)$.

We are interested in trading strategies that consist of integer positions in the risky assets. All trading strategies we consider are self-financing.

Definition 2.2 (i) An integer strategy is a predictable process $\left(\bar{\varphi}_{t}\right)_{t \in \mathbb{T} \backslash\{0\}}$ with values in $\mathbb{R} \times \mathbb{Z}^{d}$ and $\bar{\varphi}_{t} \bar{S}_{t}=\bar{\varphi}_{t+1} \bar{S}_{t}$ for $t \in \mathbb{T} \backslash\{0, T\}$. For convenience, we sometimes use the notation $\bar{\varphi}_{0}:=\bar{\varphi}_{1}$. The set of integer trading strategies is denoted by $\mathcal{Z}$.

(ii) Analogously, we define the set $\mathcal{R}$ of all (real) trading strategies and the set $\mathcal{Q}$ of rational strategies, with values in $\mathbb{R} \times \mathbb{Q}^{d}$. 
(iii) The components of a trading strategy $\bar{\varphi} \in \mathcal{R}$ are denoted by

$$
\bar{\varphi}=\left(\varphi^{0}, \ldots, \varphi^{d}\right)=\left(\varphi^{0}, \varphi\right),
$$

where we write $\varphi=\left(\varphi^{1}, \ldots, \varphi^{d}\right)$ for the risky positions.

We obviously have $\mathcal{Z} \subseteq \mathcal{Q} \subseteq \mathcal{R}$. For any trading strategy $\bar{\varphi} \in \mathcal{R}$, we denote its value at time $t \in \mathbb{T}$ by

$$
V_{t}(\bar{\varphi}):=\bar{\varphi}_{t} \bar{S}_{t}=\sum_{j=0}^{d} \bar{\varphi}_{t}^{j} S_{t}^{j},
$$

and its discounted value by $\hat{V}_{t}(\bar{\varphi}):=V_{t}(\bar{\varphi}) / S_{t}^{0}$. Often it is convenient to work with discounted asset values or discounted gains which are denoted by

$$
\begin{aligned}
\hat{S}_{t} & :=\left(S_{t}^{1}, \ldots, S_{t}^{d}\right) / S_{t}^{0}, \\
\Delta \hat{S}_{t} & :=\hat{S}_{t}-\hat{S}_{t-1}
\end{aligned}
$$

for $t \in \mathbb{T}$ and $t \in \mathbb{T} \backslash\{0\}$, respectively. The discounted value process then equals

$$
\hat{V}_{t}(\bar{\varphi})=V_{0}(\bar{\varphi})+\sum_{k=1}^{t} \varphi_{k} \Delta \hat{S}_{k}, \quad t \in \mathbb{T} .
$$

Definition 2.3 (i) An integer arbitrage is a strategy $\bar{\varphi} \in \mathcal{Z}$ which is an arbitrage for the market $\bar{S}$. This means that $V_{0}(\bar{\varphi}) \leq 0, V_{T}(\bar{\varphi}) \geq 0$ and $V_{T}(\bar{\varphi}) \not \equiv 0$.

(ii) A model satisfies the no-integer-arbitrage condition (NIA) if it admits no integer arbitrage.

(iii) Define the set (a $\mathbb{Z}$-module)

$$
\mathcal{K}_{\mathbb{Z}}:=\left\{\sum_{k=1}^{T} \varphi_{k} \Delta \hat{S}_{k}: \bar{\varphi} \in \mathcal{Z}\right\}
$$

of discounted net gains realisable by integer strategies, and

$$
\mathcal{C}_{\mathbb{Z}}:=\left(\mathcal{K}_{\mathbb{Z}}-L_{+}^{0}\right) \cap L^{\infty}
$$

Under Assumption 2.1, we define the condition NIFL (no integer free lunch) as

$$
\operatorname{cl}\left(\mathcal{C}_{\mathbb{Z}}\right) \cap L_{+}^{0}=\{0\}
$$

The closure is taken with respect to the Euclidean topology, identifying $L^{\infty}$ with $\mathbb{R}^{n}$.

Clearly, NIA is weaker than the classical no-arbitrage property NA or NIFL. It turns out that the classical no-arbitrage property NA and NIFL are equivalent (for finite probability spaces); see Theorem 2.7 below. As in the classical case, existence of an arbitrage strategy implies existence of a one-period arbitrage: 
Lemma 2.4 Suppose that the market model $\bar{S}$ admits an arbitrage in $\mathcal{Z}$ (respectively $\mathcal{Q}$ ). Then there are $\bar{\varphi} \in \mathcal{Z}$ (respectively $\mathcal{Q}$ ) and $t_{0} \in \mathbb{T} \backslash\{0\}$ such that $\varphi_{t}=0$ for $t \in \mathbb{T} \backslash\left\{t_{0}\right\}$.

Proof This is shown precisely as in the classical case (see [13, Proposition 5.11]).

The following simple properties will be used often.

Lemma 2.5 (i) In the definition of integer arbitrage, the condition $\bar{\varphi} \in \mathcal{Z}$ can be replaced by $\bar{\varphi} \in \mathcal{Q}$.

(ii) In the definition of integer arbitrage, the condition $V_{0}(\bar{\varphi}) \leq 0$ can be replaced by $V_{0}(\bar{\varphi})=0$.

(iii) NIA is equivalent to

$$
\hat{V}_{T}(\bar{\varphi})-\hat{V}_{0}(\bar{\varphi}) \geq 0 \quad \Longrightarrow \quad \hat{V}_{T}(\bar{\varphi})=\hat{V}_{0}(\bar{\varphi})
$$

for any $\bar{\varphi} \in \mathcal{Z}$ (or, equivalently, for any $\bar{\varphi} \in \mathcal{Q})$.

Proof (i) Clearly, any arbitrage strategy in $\mathcal{Z}$ is also in $\mathcal{Q}$. Now assume that there is an arbitrage in $\mathcal{Q}$. Then there exists a one-period arbitrage $\bar{\varphi} \in \mathcal{Q}$ as in Lemma 2.4. The random variable

$$
N:=\min \left\{n \in \mathbb{N}: n \varphi_{t_{0}} \in \mathbb{Z}^{d}\right\}
$$

is $\mathcal{F}_{t_{0}-1}$-measurable, and $\bar{\psi}:=N \bar{\varphi}$ is an arbitrage in $\mathcal{Z}$. Claims (ii) and (iii) are proved as in the classical case.

By (2.1), the implication (2.2) can be written as

$$
\sum_{k=1}^{T} \varphi_{k} \Delta \hat{S}_{k} \geq 0 \Longrightarrow \sum_{k=1}^{T} \varphi_{k} \Delta \hat{S}_{k}=0 .
$$

We now discuss approximation of real trading strategies by rational (respectively integer) strategies. Under Assumption 2.1 (more generally, for bounded asset price processes), the value process of any real strategy can be approximated using a rational strategy. The second part of Lemma 2.6 provides an approximation of scaled real trading strategies by integer strategies, where the error is controlled by the (integer) scaling factor. The proof is based on Dirichlet's approximation theorem (Theorem A.1 in the Appendix).

Lemma 2.6 (i) If $S$ is bounded, then for any strategy $\bar{\varphi} \in \mathcal{R}$ and any $\epsilon>0$, we can find a strategy $\bar{\psi} \in \mathcal{Q}$ such that

$$
\sup _{t \in \mathbb{T}} \operatorname{ess} \sup \left|V_{t}(\bar{\varphi})-V_{t}(\bar{\psi})\right|<\epsilon
$$

and $V_{0}(\bar{\varphi})=V_{0}(\bar{\psi})$. 
(ii) Under Assumption 2.1, let $\bar{\varphi} \in \mathcal{R}$ and $\epsilon>0$. Then there are $q \in \mathbb{N}$ and $a$ strategy $\bar{\psi} \in \mathcal{Z}$ such that $V_{0}(\bar{\varphi})=V_{0}(\bar{\psi}) / q$ and

$$
\sup _{\substack{t \in \mathbb{T}, j=1, \ldots, d, \ell=1, \ldots, n}}\left|\psi_{t}^{j}\left(\omega_{\ell}\right)-q \varphi_{t}^{j}\left(\omega_{\ell}\right)\right|<q^{-1 /(n d(T+1))}<\epsilon .
$$

In particular, for any strategy $\bar{\varphi} \in \mathcal{R}$, we can find strategies $\bar{\psi} \in \mathcal{Q}, \bar{\eta} \in \mathcal{Z}$ and $q \in \mathbb{N}$ such that

$$
\begin{gathered}
\sup _{t \in \mathbb{T}} \operatorname{ess} \sup \left|V_{t}(\bar{\varphi})-V_{t}(\bar{\psi})\right|<\epsilon, \\
\sup _{t \in \mathbb{T}} \operatorname{ess} \sup \left|q V_{t}(\bar{\varphi})-V_{t}(\bar{\eta})\right|<\epsilon,
\end{gathered}
$$

and $V_{0}(\bar{\varphi})=V_{0}(\bar{\psi})=V_{0}(\bar{\eta}) / q$

Proof Claim (i) is trivial, as any real number can be approximated by rational numbers. Thus we find a sequence of strategies $\left(\bar{\psi}^{(k)}\right)_{k \in \mathbb{N}}$ in $\mathcal{Q}$ such that $\psi^{(k)} \rightarrow \varphi$ uniformly in $\omega$ for $k \rightarrow \infty$. This and the boundedness of $S$ imply the convergence of the value at any time $t \in \mathbb{T}$ if the initial value is being fixed as equal.

To show (ii), let

$$
R_{t}:=\left\{\varphi_{t}^{j}\left(\omega_{\ell}\right): j=1, \ldots, d, \ell=1, \ldots, n\right\}
$$

For any $t \in \mathbb{T}$, let $a_{t}^{1}, \ldots, a_{t}^{K_{t}}$ be an enumeration of the elements of $R_{t}$. We have $K_{t} \leq d n$ for any $t \in \mathbb{T}$ and thus $\sum_{t \in \mathbb{T}} K_{t} \leq n d(1+T)$. By Dirichlet's approximation theorem (Theorem A.1), we find $q \in \mathbb{N}$ with $q^{-1 /(n d(1+T))}<\epsilon$ and $p_{t}^{k} \in \mathbb{Z}$ with $\left|p_{t}^{k}-q a_{t}^{k}\right|<q^{-1 /(n d(1+T))}$ for any $t \in \mathbb{T}, k=1, \ldots, K_{t}$. For $t \in \mathbb{T}$, we define

$$
\psi_{t}^{j}\left(\omega_{\ell}\right):=p_{t}^{k}
$$

where $k \in\left\{1, \ldots, K_{t}\right\}$ is such that $\varphi_{t}^{j}\left(\omega_{\ell}\right)=a_{t}^{k}$. Then

$$
\left\{\psi_{t}^{j}=p_{t}^{k}\right\} \subseteq \bigcup_{m \in A_{k}}\left\{\varphi_{t}^{j}=a_{t}^{m}\right\}
$$

where $A_{k}:=\left\{m=1, \ldots, K_{t}: p_{t}^{m}=p_{t}^{k}\right\}$. Thus $\psi_{t}$ is measurable with respect to the $\sigma$-algebra generated by $\varphi_{t}$, and hence $\mathcal{F}_{t-1}$-measurable. Therefore, $\psi$ is a predictable $\mathbb{Z}^{d}$-valued process. The uniform distance of $\psi$ and $\varphi$ is less than $q^{-1 /(n d(1+T))}$ by construction.

With the previous lemma at hand, we can show that under the finiteness condition on $\Omega$, classical no-arbitrage is equivalent to NIFL. For models of dimension $d=1$, NIA is equivalent as well, and so most of our examples will feature two-dimensional models. 
Theorem 2.7 Let Assumption 2.1 hold. Then the following statements are equivalent:

(i) There is an equivalent martingale measure $Q \approx P$.

(ii) The model satisfies the classical no-arbitrage property NA.

(iii) The model satisfies NIFL.

Moreover, if the number of risky assets is $d=1$, then the following statement is equivalent as well:

(iv) The model satisfies NIA.

Proof The equivalence of (i) and (ii) is the classical FTAP; see [13, Theorem 5.16]. Furthermore, NA is equivalent to the classical no-free-lunch condition in our setup (see $[9,17]$ ), which yields the implication (ii) $\Rightarrow$ (iii).

Now we assume (iii) and show (ii). Let $\bar{\varphi} \in \mathcal{R}$ be such that $V_{0}(\bar{\varphi})=0$ and $V_{T}(\bar{\varphi}) \geq 0$. By part (ii) of Lemma 2.6, we find $q_{N} \in \mathbb{N}$ and strategies $\bar{\psi}^{(N)} \in \mathcal{Z}$ such that

$$
\operatorname{ess} \sup \left|q_{N} V_{T}(\bar{\varphi})-V_{T}\left(\bar{\psi}^{(N)}\right)\right| \leq \frac{1}{N} .
$$

Without loss of generality, the sequence $\left(q_{N}\right)$ increases (not necessarily strictly). We get

$$
V_{T}\left(\bar{\psi}^{(N)}\right) \geq q_{N} V_{T}(\bar{\varphi})-\frac{1}{N} \geq-\frac{1}{N}
$$

Define

$$
\begin{aligned}
Z_{N} & :=\min \left(1, V_{T}\left(\bar{\psi}^{(N)}\right)\right) \\
& =V_{T}\left(\bar{\psi}^{(N)}\right)-\left(V_{T}\left(\bar{\psi}^{(N)}\right)-1\right) 1_{\left\{V_{T}\left(\bar{\psi}^{(N)}\right)>1\right\}} \in \mathcal{C}_{\mathbb{Z}}, \quad N \in \mathbb{N} .
\end{aligned}
$$

Since $Z_{N} \in[-1 / N, 1]$, there is a convergent subsequence, and without loss of generality, $\left(Z_{N}\right)$ itself converges to some $Z \in \operatorname{cl}\left(\mathcal{C}_{\mathbb{Z}}\right)$. By (2.4), we have $Z \geq 0$. Then NIFL implies that $Z=0$, and thus $V_{T}\left(\bar{\psi}^{(N)}\right) \rightarrow 0$. Since $q_{N}^{-1} V_{T}\left(\bar{\psi}^{(N)}\right) \rightarrow V_{T}(\bar{\varphi})$ by $(2.3)$ (recall that $\left(q_{N}\right)$ increases), we conclude that $V_{T}(\bar{\varphi})=0$.

For the final part, (ii) $\Rightarrow$ (iv) is obvious. Now assume that $d=1$ and that (ii) does not hold. Lemma 2.4 yields the existence of a one-period arbitrage, i.e., an arbitrage $\bar{\varphi}$ and $t_{0} \in \mathbb{T}$ such that $\varphi_{t}=0$ for any $t \in \mathbb{T} \backslash\left\{t_{0}\right\}$. Since $\bar{\varphi}$ is an arbitrage, we must have $\varphi_{t_{0}}^{1} \neq 0$. Define

$$
\psi_{t}^{j}:=1_{\left\{\varphi_{t_{0}}^{1} \neq 0\right\}} \frac{\varphi_{t}^{j}}{\left|\varphi_{t_{0}}^{1}\right|}, \quad t \in \mathbb{T}, j=0,1 .
$$

Then $\bar{\psi}$ is an arbitrage as well. Moreover, $\psi_{t}^{1} \in\{-1,0,1\} \subseteq \mathbb{Z}$ for any $t \in \mathbb{T}$, thus $\bar{\psi} \in \mathcal{Z}$. Consequently, (iv) does not hold.

In practice, all values occurring in the model specification are floating-point numbers. The following result shows that in this case, the existence of an arbitrage opportunity is not affected by integer constraints. 
Theorem 2.8 Let Assumption 2.1 hold, and assume that the interest rate $r$ and all asset values are rational, i.e., $r \in \mathbb{Q}$ and $S_{t} \in \mathbb{Q}^{d}$ for $t \in \mathbb{T}$. Then NIA is equivalent to NA.

Proof NA always implies NIA. Now suppose that we have a real arbitrage opportunity. By part (iii) of Lemma 2.5, there is a predictable process $\varphi$ such that

$$
\begin{aligned}
& \forall \omega \in \Omega: \sum_{k=1}^{T} \varphi_{k}(\omega) \Delta \hat{S}_{k}(\omega) \geq 0, \\
& \exists \omega \in \Omega: \sum_{k=1}^{T} \varphi_{k}(\omega) \Delta \hat{S}_{k}(\omega)>0 .
\end{aligned}
$$

The assertion now follows from Lemma 2.9 below. Note that predictability of the resulting rational process is easy to guarantee by introducing for all $k, j$ a single variable for all the $\varphi_{k}^{j}(\omega)$ for which $\omega$ belongs to the same atom of $\mathcal{F}_{k-1}$.

In the proof of the preceding result, we have applied the following simple lemma. Using Ehrhart's theory of lattice points in dilated polytopes [4, Chap. 3], [21, Sect. 4.6], it is certainly possible to state much more general results along these lines. (We thank Manuel Kauers for pointing this out.) Therefore, we do not claim originality for Lemma 2.9, but give a short self-contained proof for the reader's convenience.

Lemma 2.9 Let $\left(a_{i j}\right)_{1 \leq i \leq r, 1 \leq j \leq s}$ be a matrix with rational entries $a_{i j} \in \mathbb{Q}$. Suppose that there is a real vector $\left(x_{1}, \ldots, x_{s}\right)$ such that

$$
\sum_{j=1}^{s} a_{i j} x_{j} \geq 0, \quad i=1, \ldots, r, \text { with at least one inequality being strict }
$$

Then there is a rational vector satisfying (2.5).

Proof After possibly reordering the rows of the matrix $\left(a_{i j}\right)$, we may assume that there is $u \in\{1, \ldots, r\}$ such that

$$
\begin{array}{ll}
\sum_{j=1}^{s} a_{i j} x_{j}>0, & 1 \leq i \leq u, \\
\sum_{j=1}^{s} a_{i j} x_{j}=0, & u<i \leq r .
\end{array}
$$

By defining $y_{i}:=\sum_{j=1}^{s} a_{i j} x_{j}$ for $1 \leq i \leq u$, we get that the vector

$$
\left(x_{1}, \ldots, x_{s}, y_{1}, \ldots, y_{u}\right)
$$


solves the system

$$
\begin{aligned}
& \sum_{j=1}^{s} a_{i j} x_{j}-y_{i}=0, \quad 1 \leq i \leq u, \\
& \sum_{j=1}^{s} a_{i j} x_{j}=0, \quad u<i \leq r, \\
& y_{1}, \ldots, y_{u}>0 \text {. }
\end{aligned}
$$

Equations (2.6) and (2.7) constitute a homogeneous linear system of equations with rational coefficients, which has a basis $B \subseteq \mathbb{Q}^{s+u}$ of rational solution vectors, by Gauss elimination. The vector $\left(x_{1}, \ldots, x_{s}, y_{1}, \ldots, y_{u}\right)$ can be written as a linear combination of vectors in $B$. By approximating the (real) coefficients of this linear combination with rational numbers, we get a vector $\left(\tilde{x}_{1}, \ldots, \tilde{x}_{s}, \tilde{y}_{1}, \ldots, \tilde{y}_{u}\right) \in \mathbb{Q}^{s+u}$ satisfying (2.6)-(2.8). Then $\left(\tilde{x}_{1}, \ldots, \tilde{x}_{s}\right)$ is the desired rational vector.

The assertion of Theorem 2.8 does not hold for infinite probability spaces, as the following example illustrates.

Example 2.10 Let $\Omega=\left\{\omega_{1}, \omega_{2}, \ldots\right\}$ be countable, $\mathcal{A}=2^{\Omega}$, and fix an arbitrary probability measure $P$ with $P\left[\left\{\omega_{i}\right\}\right]>0$ for all $i \in \mathbb{N}$. We choose $d=2, T=1$ and $r=0$. The asset prices are defined by $S_{0}=(1,1)$ and

$$
S_{1}\left(\omega_{i}\right)= \begin{cases}\left(1+p_{i}, 1+q_{i}\right), & i \text { even, } \\ \left(1-\hat{p}_{i}, 1-\hat{q}_{i}\right), & i \text { odd }\end{cases}
$$

where $p_{i}, q_{i}, \hat{p}_{i}, \hat{q}_{i}$ are natural numbers satisfying

$$
\frac{p_{i}}{q_{i}} \searrow \pi \quad \text { and } \quad \frac{\hat{p}_{i}}{\hat{q}_{i}} \nearrow \pi, \quad i \rightarrow \infty .
$$

Thus the increments are

$$
\Delta S_{1}\left(\omega_{i}\right)= \begin{cases}\left(p_{i}, q_{i}\right), & i \text { even } \\ \left(-\hat{p}_{i},-\hat{q}_{i}\right), & i \text { odd }\end{cases}
$$

A vector $\left(\varphi_{1}^{1}, \varphi_{1}^{2}\right) \in \mathbb{R}^{2}$ yields an arbitrage if and only if

$$
\begin{array}{ll}
\frac{p_{i}}{q_{i}} \varphi_{1}^{1}+\varphi_{1}^{2} \geq 0, & i \text { even, } \\
\frac{\hat{p}_{i}}{\hat{q}_{i}} \varphi_{1}^{1}+\varphi_{1}^{2} \leq 0, & i \text { odd },
\end{array}
$$

with at least one inequality being strict. By (2.9), the vector $\left(\varphi_{1}^{1}, \varphi_{1}^{2}\right)=(1,-\pi)$ satisfies this, and so NA does not hold. By letting $i \rightarrow \infty$, we see that there is no integer vector satisfying (2.10) and (2.11), which shows that the model satisfies NIA. 
Our next goal is to characterise NIA without restricting the asset prices to the rational numbers. As we shall see, for $d>1$, NIA is not equivalent to the existence of an equivalent martingale measure, but rather to the existence of an absolutely continuous martingale measure with an additional property. We first introduce sets of strategies which do not yield any net profit.

Definition 2.11 (i) Let $Q$ be a probability measure on $(\Omega, \mathcal{A})$, and denote the set of trading strategies with zero initial value by

$$
\mathcal{R}_{0}:=\left\{\bar{\varphi} \in \mathcal{R}: V_{0}(\bar{\varphi})=0\right\}
$$

We denote the set of all integer-valued (resp. rational-valued, resp. real-valued) trading strategies with zero initial capital and $Q$-a.s. zero gain by

$$
\begin{aligned}
& \mathcal{Z}_{Q}^{0}:=\left\{\bar{\varphi} \in \mathcal{R}_{0} \cap \mathcal{Z}: V_{T}(\bar{\varphi})=0 Q \text {-a.s. }\right\}, \\
& \mathcal{Q}_{Q}^{0}:=\left\{\bar{\varphi} \in \mathcal{R}_{0} \cap \mathcal{Q}: V_{T}(\bar{\varphi})=0 Q \text {-a.s. }\right\}, \\
& \mathcal{R}_{Q}^{0}:=\left\{\bar{\varphi} \in \mathcal{R}_{0}: V_{T}(\bar{\varphi})=0 Q \text {-a.s. }\right\}
\end{aligned}
$$

(ii) Under Assumption 2.1, we write $\mathfrak{Q}_{\mathbb{Z}}^{\max }$ for the set of martingale measures $Q \ll P$ such that

$$
\begin{aligned}
& \forall \bar{\varphi} \in \mathcal{Q}_{Q}^{0}: V_{T}(\bar{\varphi}) \geq 0 \Rightarrow V_{T}(\bar{\varphi})=0, \quad \text { and } \\
& \exists \bar{\varphi} \in \mathcal{R}_{Q}^{0}: V_{T}(\bar{\varphi}) \geq 0 \text { and }\left\{V_{T}(\bar{\varphi})>0\right\}=\{\omega \in \Omega: Q[\{\omega\}]=0\}
\end{aligned}
$$

(iii) $\mathfrak{Q}_{\mathbb{Z}}$ denotes the set of martingale measures $Q \ll P$ such that

$$
\forall \bar{\varphi} \in \mathcal{Z}_{Q}^{0}: V_{T}(\bar{\varphi}) \geq 0 \Rightarrow V_{T}(\bar{\varphi})=0
$$

Note that Assumption 2.1 ensures that the sets $\{\omega\}$ occurring in (ii) are measurable. Obviously, we have $\mathfrak{Q} \subseteq \mathfrak{Q}_{\mathbb{Z}}^{\max } \subseteq \mathfrak{Q}_{\mathbb{Z}}$, where $\mathfrak{Q}$ denotes the set of equivalent martingale measures. (For the first inclusion, $\bar{\varphi}=0$ satisfies the existence statement in (ii).) The existence statement in (ii) means that there is a real arbitrage which realises a gain on the complement of the support of the measure. This means in particular that there is a cone $C$ in $\mathbb{R}^{|\Omega| \times \mathbb{T}}$ which corresponds to real arbitrage strategies. The "for all" statement in (ii) asserts that none of these real arbitrages lies in $\mathcal{Z}$, i.e., $C \cap \mathbb{Z}^{|\Omega| \times \mathbb{T}}=\emptyset$.

Before giving an FTAP for integer trading, we show further properties of the measures in $\mathfrak{Q}_{\mathbb{Z}}^{\max }$.

Proposition 2.12 Let Assumption 2.1 hold, and assume that $\mathfrak{Q}_{\mathbb{Z}}^{\max } \neq \emptyset$. Then there is a set $A \subsetneq \Omega$ such that $\mathfrak{Q}_{\mathbb{Z}}^{\max }$ is the set of martingale measures whose support is $\Omega \backslash A$. Also, there is $\bar{\varphi} \in \mathcal{R}_{0}$ with $V_{T}(\bar{\varphi}) \geq 0$ and $\left\{V_{T}(\bar{\varphi})>0\right\}=A$.

Now let $Q \in \mathfrak{Q}_{\mathbb{Z}}^{\max }$ and $Q^{\prime} \in \mathfrak{Q}_{\mathbb{Z}}$. Then $Q^{\prime} \ll Q$. Moreover, $\mathfrak{Q}_{\mathbb{Z}}^{\max }$ is dense in $\mathfrak{Q}_{\mathbb{Z}}$ with respect to the total variation distance. 
Proof Choose $Q \in \mathfrak{Q}_{\mathbb{Z}}^{\max }$ and let $\bar{\varphi} \in \mathcal{R}_{Q}^{0}$ satisfy the existence statement in (ii) of Definition 2.11. Define $A:=\left\{V_{T}(\bar{\varphi})>0\right\}$. Then $\bar{\varphi}$ is the required trading strategy.

Let $Q^{\prime}$ be a martingale measure with support equal to $\Omega \backslash A$. Then $\bar{\varphi}$ satisfies the existence statement of (ii) in Definition 2.11. Let $\bar{\psi} \in \mathcal{Q}_{Q^{\prime}}^{0}$ with $V_{T}(\bar{\psi}) \geq 0$. Then $V_{T}(\bar{\psi})=0 Q^{\prime}$-a.s., i.e., $V_{T}(\bar{\psi})=0$ on $\Omega \backslash A$. Consequently, $\bar{\psi} \in \mathcal{Q}_{Q}^{0}$. Part (ii) of Definition 2.11 yields that $V_{T}(\bar{\psi})=0$. Thus, $Q^{\prime} \in \mathfrak{Q}_{\mathbb{Z}}^{\max }$.

We need to show that any measure in $\mathfrak{Q}_{\mathbb{Z}}^{\max }$ is a martingale measure with support $\Omega \backslash A$. This, however, follows as soon as we have shown that $Q^{\prime} \ll Q$ for any $Q^{\prime} \in \mathfrak{Q}_{\mathbb{Z}}$. Let $Q^{\prime} \in \mathfrak{Q}_{\mathbb{Z}}$. Observe that $V_{T}(\bar{\varphi})=0 Q^{\prime}$-a.s. because $Q^{\prime}$ is a martingale measure. Thus $A=\left\{V_{T}(\bar{\varphi})>0\right\}$ is a $Q^{\prime}$-null set. We find $Q^{\prime} \ll Q$.

Finally, we have to show that $Q^{\prime}$ can be approximated by elements in $\mathfrak{Q}_{\mathbb{Z}}^{\max }$ in total variation. Define $Q_{\alpha}:=\alpha Q^{\prime}+(1-\alpha) Q$ for any $\alpha \in[0,1]$. Then $Q_{\alpha} \rightarrow Q_{1}=Q^{\prime}$ as $\alpha \rightarrow 1$. However, $Q_{\alpha}$ is a martingale measure with the same support as $Q$ for $\alpha \neq 1$, and hence it is in $\mathfrak{Q}_{\mathbb{Z}}^{\max }$ by what we have shown so far.

We can now state an FTAP for integer trading.

Theorem 2.13 Under Assumption 2.1, the following statements are equivalent:

(i) $\mathfrak{Q}_{\mathbb{Z}}^{\max } \neq \varnothing$.

(ii) $\mathfrak{Q}_{\mathbb{Z}} \neq \emptyset$.

(iii) The market satisfies NIA.

The implication (ii) $\Rightarrow$ (iii) does not need Assumption 2.1 .

Proof The implication (i) $\Rightarrow$ (ii) is trivial.

(ii) $\Rightarrow$ (iii). Fix a measure $Q \in \mathfrak{Q}_{\mathbb{Z}}$. Let $\bar{\varphi} \in \mathcal{R}_{0} \cap \mathcal{Z}$ with $V_{T}(\bar{\varphi}) \geq 0$. Since $Q$ is a martingale measure, we have $\hat{V}_{T}(\bar{\varphi})=0 Q$-a.s. and hence $V_{T}(\bar{\varphi})=0 Q$-a.s. Thus $\bar{\varphi} \in \mathcal{Z}_{Q}^{0}$. By part (iii) of Definition 2.11, we have $V_{T}(\bar{\varphi})=0$. Hence we have NIA.

(iii) $\Rightarrow$ (i). Let

$$
A:=\left\{\omega \in \Omega: \exists \bar{\varphi} \in \mathcal{R}_{0} \text { with } V_{T}(\bar{\varphi}) \geq 0 \text { and } V_{T}(\bar{\varphi})(\omega)>0\right\} .
$$

For every $\omega \in A$, choose a corresponding strategy $\bar{\varphi}^{(\omega)} \in \mathcal{R}_{0}$ with $V_{T}\left(\bar{\varphi}^{(\omega)}\right) \geq 0$ and $V_{T}\left(\bar{\varphi}^{(\omega)}\right)(\omega)>0$. Define

$$
\bar{\varphi}:=\sum_{\omega \in A} \bar{\varphi}^{(\omega)} .
$$

Then $\bar{\varphi} \in \mathcal{R}_{0}, V_{T}(\bar{\varphi}) \geq 0$ and $\left\{V_{T}(\bar{\varphi})>0\right\}=A$.

We claim that for any $\bar{\psi} \in \mathcal{R}_{0}$ with $V_{T}(\bar{\psi}) 1_{\Omega \backslash A} \geq 0$, we have $V_{T}(\bar{\psi}) 1_{\Omega \backslash A}=0$. Let $\bar{\psi} \in \mathcal{R}_{0}$ with $V_{T}(\bar{\psi}) 1_{\Omega \backslash A} \geq 0$. If $V_{T}(\bar{\psi}) \geq 0$ on $A$, then $V_{T}(\bar{\psi}) \geq 0$ and hence $V_{T}(\bar{\psi}) 1_{\Omega \backslash A}=0$ by the construction of $A$. Thus we may assume that $V_{T}(\bar{\psi})(\omega)<0$ for some $\omega \in A$. Then

$$
c:=-\frac{\min \left\{V_{T}(\bar{\psi})(\omega): \omega \in A\right\}}{\min \left\{V_{T}(\bar{\varphi})(\omega): \omega \in A\right\}}>0 .
$$

The strategy $\bar{\psi}+c \bar{\varphi}$ is in $\mathcal{R}_{0}$ and satisfies $V_{T}(\bar{\psi}+c \bar{\varphi}) \geq 0$. Thus $V_{T}(\bar{\psi}+c \bar{\varphi})=0$ outside of $A$. Hence $V_{T}(\bar{\psi})=0$ outside of $A$, i.e., $V_{T}(\bar{\psi}) 1_{\Omega \backslash A}=0$. 
Assume for a contradiction that $A=\Omega$. Then $V_{T}(\bar{\varphi})>0$. Define

$$
e:=\min \left\{V_{T}(\bar{\varphi})(\omega): \omega \in \Omega\right\}>0 .
$$

Lemma 2.6 yields $q \in \mathbb{N}$ and $\bar{\psi} \in \mathcal{Z}$ such that $\left|V_{T}(\bar{\psi})-V_{T}(q \bar{\varphi})\right|<e$. Thus $V_{T}(\bar{\psi})>q V_{T}(\bar{\varphi})-e \geq 0$. Thus $\bar{\psi}$ is an integer arbitrage, which is a contradiction.

Consequently, $A \subsetneq \Omega$. We have shown that the market $\bar{S}$ is free of arbitrage on $\Omega \backslash A$. (This means that restricting the process $\bar{S}$ to $\Omega \backslash A$ yields an arbitrage-free model, where the $\sigma$-algebra on the underlying probability space $\Omega \backslash A$ is the trace $\sigma$-algebra.) The classical fundamental theorem yields a martingale measure $Q$ on $\Omega \backslash A$ for $\bar{S}$. We denote its extension to a probability measure on $\Omega$ by $Q$, i.e., $Q[M]=Q[M \cap(\Omega \backslash A)]$ for any $M \subseteq \Omega$. Then $Q \ll P$ and $Q$ is a martingale measure with $\{\omega \in \Omega: Q[\{\omega\}]=0\}=A$. Since $\left\{V_{T}(\bar{\varphi})>0\right\}=A$, we have the existence statement in part (ii) of Definition 2.11. Now let $\bar{\psi} \in \mathcal{Q}_{Q}^{0}$ with $\hat{V}_{T}(\bar{\psi}) \geq 0$. Let $q$ be a common denominator for

$$
\left\{\psi_{t}^{j}\left(\omega_{\ell}\right): t \in \mathbb{T}, j=1, \ldots, d, \ell=1, \ldots, n\right\}
$$

Then $q \bar{\psi} \in \mathcal{Z}_{Q}^{0}$. Since we have NIA, we get $V_{T}(\bar{\psi})=\frac{1}{q} V_{T}(q \bar{\psi})=0$ as claimed.

An immediate consequence is the following sufficient criterion for the construction of markets with no integer arbitrage.

Corollary 2.14 Let $Q \ll P$ be a martingale measure and assume that $\mathcal{Z}_{Q}^{0}=\{0\}$. Then the market satisfies NIA.

The following example is a simple application of the preceding corollary. Here and in all examples that follow, Assumption 2.1 is in force.

Example 2.15 Assume that $d=2, n \geq 2, T=1, r=0$ and choose $\left(S_{0}^{1}, S_{0}^{2}\right)=(1, \pi)$ and

$$
\left(S_{1}^{1}, S_{1}^{2}\right)\left(\omega_{j}\right):= \begin{cases}(3 / 2,3 \pi / 2), & j=1, \\ (1 / 2, \pi / 2), & j=2 .\end{cases}
$$

Define $Q\left[\left\{\omega_{j}\right\}\right]=1_{\{j=1,2\}} / 2$ for $j=1, \ldots, n$. Then $Q \ll P$ is a martingale measure, and $\mathcal{R}_{Q}^{0}=\left\{\left(0,-\pi \varphi^{2}, \varphi^{2}\right): \varphi^{2} \in \mathbb{R}\right\}$. Consequently, we have $\mathcal{Z}_{Q}^{0}=\{0\}$. Thus Corollary 2.14 yields that the market does not allow integer arbitrage. Observe that this holds regardless of the specification of $\left(S_{1}^{1}, S_{1}^{2}\right)\left(\omega_{j}\right)$ for $j \geq 3$.

Another immediate consequence is the existence of absolutely continuous martingale measures.

Corollary 2.16 Suppose that a model satisfies NIA and let Assumption 2.1 hold. Then there is an absolutely continuous martingale measure.

Proof Immediate from Theorem 2.13 , (iii) $\Rightarrow$ (i). 
The following example shows that the existence of an absolutely continuous martingale measure alone is insufficient to exclude integer arbitrage.

Example 2.17 Let $\Omega=\left\{\omega_{1}, \omega_{2}\right\}, S_{0}^{0}=1=S_{1}^{0}, S_{0}^{1}=1$ and $S_{1}^{1}\left(\omega_{i}\right)=i$ for $i=1,2$ (i.e., $T=1, d=1, n=2$ ). Then $Q:=\delta_{\omega_{1}}$ is a martingale measure which is absolutely continuous with respect to $P:=\left(\delta_{\omega_{1}}+\delta_{\omega_{2}}\right) / 2$, where $\delta_{\omega_{j}}$ denotes the Diracmeasure on $\omega_{j}$. The strategy $\bar{\varphi}_{1}:=(-1,1)$ is an integer arbitrage.

Finally, we provide a technical statement that will be used in Sect. 4 .

Lemma 2.18 Let Assumption 2.1 hold, let $Q \in \mathfrak{Q}_{\mathbb{Z}}^{\max }$, and assume that for any set $B \in \mathcal{F}_{1}$, we have $Q[B] \in\{0,1\}$. Then $\mathcal{F}_{1}=\mathcal{F}_{0}$.

Proof Let $A \in \mathcal{F}_{1}$ be maximal with $Q[A]=0$. Then $B:=\Omega \backslash A$ is an atom, and its only strict subset contained in $\mathcal{F}_{1}$ is the empty set. If $A=\emptyset$, the claim follows trivially. Assume for a contradiction that $A \neq \emptyset$. We claim that the model restricted to $A$ still satisfies NIA. To this end, let $\bar{\varphi} \in \mathcal{Q}$ with $\bar{\varphi}_{s}=0$ on $A$ for $1 \leq s<t$ and $\hat{V}_{t}(\bar{\varphi})=\cdots=\hat{V}_{T}(\bar{\varphi}) \geq 0$ on $A$, for some $t \in\{1, \ldots, T\}$. Since existence of an arbitrage implies existence of a one-period arbitrage, see Lemma 2.4, it suffices to consider this kind of strategy and show that there is no arbitrage in the $t$ th period.

Case 1: $t=1$. Any random variable $X$ is a.s. constant on the atom $B$, and we write $X(B)$ for this value. Since $\hat{S}_{0}=E_{Q}\left[\hat{S}_{1}\right]=\hat{S}_{1}(B)$, we find that

$$
\hat{V}_{1}(\bar{\varphi})(B)=V_{0}(\bar{\varphi})=0,
$$

and hence $V_{1}(\bar{\varphi}) \geq 0$ everywhere. As $\left(S^{0}, \ldots, S^{d}\right)$ satisfies NIA (on $\Omega$ ), we obtain that $V_{1}(\bar{\varphi})=0$.

Case 2: $t \geq 2$. Define $\bar{\psi}:=1_{A} \bar{\varphi}$. Since $A \in \mathcal{F}_{1}$ and $\bar{\varphi}_{1}=0$, we find that $\bar{\psi} \in \mathcal{Q}$ with $\bar{\psi}_{0}=\cdots=\bar{\psi}_{t-1}=0$ and $\hat{V}_{t}(\bar{\psi})=\cdots=\hat{V}_{T}(\bar{\psi}) \geq 0$. Because the model $\left(S^{0}, \ldots, S^{d}\right)$ on $\Omega$ satisfies NIA by assumption, we find that

$$
0=V_{t}(\bar{\psi})=1_{A} V_{t}(\bar{\varphi})
$$

and hence $V_{t}(\bar{\varphi})=0$ on $A$. Thus $\left(S^{0}, \ldots, S^{d}\right)$ restricted to $A$ satisfies NIA. By Theorem 2.13, there is $Q^{\prime} \in \mathfrak{Q}_{\mathbb{Z}}^{\max }$ for the model $\left(S^{0}, \ldots, S^{d}\right)$ restricted to $A$. We denote its extension to $\Omega$ by $Q^{\prime}$ as well, i.e., $Q^{\prime}[C]=Q^{\prime}[C \cap A]$ for any $C \in \mathcal{A}$. Define $\tilde{Q}:=Q / 2+Q^{\prime} / 2$ and observe that $\tilde{Q} \in \mathfrak{Q}_{\mathbb{Z}}$. However, $Q \not \approx \tilde{Q}$ because $Q^{\prime}$ has disjoint support with $Q$. But Proposition 2.12 implies $Q \approx \tilde{Q}$, which yields a contradiction. Thus $A=\emptyset$ and hence $\mathcal{F}_{1}=\mathcal{F}_{0}$.

\section{Claims and integer trading}

Definition 3.1 Fix a model that satisfies NIA. 
(i) A claim is an $\mathcal{F}_{T}$-measurable random variable $C \geq 0$. A real number $p \geq 0$ is an integer-arbitrage-free price of $C$ if there is an adapted nonnegative stochastic process $\left(X_{t}\right)_{t \in \mathbb{T}}$ with $X_{0}=p, X_{T}=C$ such that the market

$$
\left(S^{0}, \ldots, S^{d}, X\right)
$$

satisfies NIA. The set of integer-arbitrage-free prices is denoted by $\Pi_{\mathbb{Z}}(C)$.

(ii) An integer superhedge for $C$ is a strategy $\bar{\varphi} \in \mathcal{Z}$ such that $V_{T}(\bar{\varphi}) \geq C$, and it is an integer replication strategy if it satisfies $V_{T}(\bar{\varphi})=C$ a.s. We write

$$
\sigma_{\mathbb{Z}}(C)=\inf \left\{V_{0}(\bar{\varphi}): \bar{\varphi} \in \mathcal{Z}, V_{T}(\bar{\varphi}) \geq C\right\}
$$

for the infimum of prices of integer superreplication strategies for $C$.

Analogously to $\Pi_{\mathbb{Z}}(C)$, we write $\Pi(C)$ for the set of classical arbitrage-free prices in models satisfying NA. We recall the classical superhedging theorem (Corollaries 7.15 and 7.18 in [13]):

Theorem 3.2 Assume that NA holds, and let $C$ be a claim with $\sup \Pi(C)<\infty$. Then there is a strategy $\bar{\varphi} \in \mathcal{R}$ with $V_{0}(\bar{\varphi})=\sup \Pi(C)$ and $V_{T}(\bar{\varphi}) \geq C$. Moreover, sup $\Pi(C)$ is the smallest number with this property.

We find analogous statements to the preceding theorem under the weaker assumption NIA. Proposition 3.8 below states that NIA suffices for the existence of a real cheapest superhedge whose price is the infimum of all rational superhedging prices. Moreover, Theorem 4.3 below implies that either the set of NIA-compatible prices for the claim is empty, or its supremum equals the cheapest superhedging price.

There is no need to define the notion of integer completeness, because there would be no interesting models that have this property. Note that Assumption 2.1 is not needed for the following result.

Proposition 3.3 The following statements are equivalent:

(i) Every claim is replicable by an integer strategy.

(ii) The probability space $(\Omega, \mathcal{A}, P)$ consists of a single atom.

Proof If (ii) holds and $C$ is a claim, then there is a constant $c \in[0, \infty)$ such that $C=c$ a.s. Then $C$ is replicated by the integer strategy $\bar{\varphi}=\left(\varphi^{0}, 0\right)$ with $\varphi_{t}^{0}=c /(1+r)^{T-t}$, $t \in \mathbb{T}$.

Now suppose that every claim is integer replicable. In particular, each claim is then replicable in the classical sense. It is well known that this implies that $\Omega$ has a partition into finitely many atoms. (This result is usually proved in the framework of a model satisfying NA. Assuming NA is not necessary though, as seen from the proof of Theorem 5.37 in [13].) If $\Omega$ does not consist of a single atom, we can fix two distinct atoms $A$ and $B$. The notation $X(A)$ has the same meaning as in the proof of Lemma 2.18 . A self-financing integer trading strategy $\bar{\varphi}$ is uniquely defined by specifying its initial wealth $V_{0}(\bar{\varphi})$ and the predictable $\mathbb{Z}^{d}$-valued process $\varphi=\left(\varphi_{t}\right)_{t=1, \ldots, T}$. Thus there is a bijective map

$$
\Gamma: \mathbb{R} \times \mathcal{Z}_{\mathrm{c}} \rightarrow \mathcal{Z}
$$


where $\mathcal{Z}_{\mathrm{c}}:=\left\{\left(\varphi^{1}, \ldots, \varphi^{d}\right): \bar{\varphi} \in \mathcal{Z}\right\}$ is countable, with $V_{0}(\Gamma(v, \varphi))=v$ for any $v \in \mathbb{R}$. In particular, $v \mapsto V_{T}(\Gamma(v, \varphi))$ is affine. We have

$$
\begin{aligned}
& \left\{(a, b) \in[0, \infty)^{2}: a 1_{A}+b 1_{B} \text { can be integer replicated }\right\} \\
& \subseteq\left\{\left(V_{T}(\bar{\varphi})(A), V_{T}(\bar{\varphi})(B)\right): \bar{\varphi} \in \mathcal{Z}\right\} \\
& =\bigcup_{\varphi \in \mathcal{Z}_{\mathrm{c}}}\left\{\left(V_{T}(\Gamma(v, \varphi))(A), V_{T}(\Gamma(v, \varphi))(B)\right): v \in \mathbb{R}\right\} .
\end{aligned}
$$

For each $\varphi \in \mathcal{Z}_{\mathrm{c}}$, the set

$$
\left\{\left(V_{T}(\Gamma(v, \varphi))(A), V_{T}(\Gamma(v, \varphi))(B)\right): v \in \mathbb{R}\right\}
$$

is a nullset for two-dimensional Lebesgue measure because it is a one-dimensional affine space in $\mathbb{R}^{2}$. We conclude that (3.3) has Lebesgue measure zero, and hence (3.2) is a nullset, too. This contradicts our assumption.

Recall that in the classical case (under Assumption 2.1, so that integrability holds), the set of arbitrage-free prices has the representation

$$
\Pi(C)=\left\{E_{Q}\left[\frac{C}{(1+r)^{T}}\right]: Q \in \mathfrak{Q}\right\},
$$

where $\mathfrak{Q}$ is the set of equivalent martingale measures. The corresponding result for NIA looks as follows.

Proposition 3.4 Let Assumption 2.1 hold, and suppose that the model satisfies NIA. Let $C$ be a claim. Then

$$
\Pi_{\mathbb{Z}}(C) \subseteq\left\{E_{Q}\left[\frac{C}{(1+r)^{T}}\right]: Q \in \mathfrak{Q}_{\mathbb{Z}}\right\} .
$$

Proof Suppose that $p \in \Pi_{\mathbb{Z}}(C)$. Then there is an adapted process $X$ such that $X_{0}=p, X_{T}=C$ and $\left(S^{0}, \ldots, S^{d}, X\right)$ satisfies NIA. Let $\mathfrak{Q}_{\mathbb{Z}}$ be the set of absolutely continuous martingale measures that satisfy part (iii) of Definition 2.11 for this market. By Theorem 2.13, there is $Q \in \hat{\mathfrak{Q}}_{\mathbb{Z}} \subseteq \mathfrak{Q}_{\mathbb{Z}}$. Then $p=E_{Q}\left[\frac{C}{(1+r)^{T}}\right]$.

The following example shows that the inclusion in Proposition 3.4 can be strict. In fact, in this example, we have $\Pi_{\mathbb{Z}}(C)=\emptyset$. Note that some asset values in Example 3.5 are irrational. By Theorem 2.8, no such example can be constructed using only rational values. This also applies to all our other examples featuring irrational values.

Example 3.5 Let $\Omega=\left\{\omega_{1}, \omega_{2}, \omega_{3}\right\}, r=0, d=2, T=1$ and $P\left[\left\{\omega_{j}\right\}\right]=1 / 3$ for $j=1,2,3$. We choose $\left(S_{0}^{1}, S_{0}^{2}\right)=(\pi, 1)$ and

$$
\left(S_{1}^{1}, S_{1}^{2}\right)\left(\omega_{j}\right)= \begin{cases}(2 \pi, 2), & j=1, \\ \left(\frac{\pi}{2}, \frac{1}{2}\right), & j=2, \\ (\pi, 2), & j=3 .\end{cases}
$$


A short calculation reveals that $Q\left[\left\{\omega_{j}\right\}\right]:=1_{\{j \neq 3\}} j / 3$ is the only martingale measure. Obviously, we have $Q \ll P$, and the only integer strategy with zero initial wealth and $Q$-a.s. zero final wealth is identically zero. Thus $Q$ satisfies (ii) in Theorem 2.13, and hence we have NIA. Since $Q$ is the only martingale measure, we have $\mathfrak{Q}_{\mathbb{Z}}=\{Q\}=\mathfrak{Q}_{\mathbb{Z}}^{\max }$.

Now we consider the claim $C:=1_{\left\{\omega_{3}\right\}}$. Proposition 3.4 yields that

$$
\Pi_{\mathbb{Z}}(C) \subseteq\left\{E_{Q}[C]\right\}=\{0\} .
$$

Define the extended model $\left(S^{0}, S^{1}, S^{2}, X\right)$ with $X_{0}:=0, X_{1}:=C$. Since $(0,0,0,1)$ is an integer arbitrage for the extended model, we have $0 \notin \Pi_{\mathbb{Z}}(C)$, and so

$$
\Pi_{\mathbb{Z}}(C)=\emptyset \subsetneq\{0\}=\left\{E_{R}\left[\frac{C}{(1+r)^{T}}\right]: R \in \mathfrak{Q}_{\mathbb{Z}}\right\} .
$$

In Sect. 4, we shall see that $\Pi_{\mathbb{Z}}(C)$ is either empty or dense in the right-hand side of (3.4).

If the model satisfies NA (and not just NIA), we can compare the sets of classical, resp. integer-arbitrage-free, prices. Theorem 5.32 in [13] states that $\Pi(C)$ is an open interval if $C$ is non-replicable, and a singleton if $C$ is replicable. It turns out that under NA, the set $\Pi_{\mathbb{Z}}(C)$ is an interval, too, which may differ from $\Pi(C)$ only at the endpoints. In particular, if NA holds, then $\Pi_{\mathbb{Z}}(C)$ cannot be empty.

Theorem 3.6 Let Assumption 2.1 hold, suppose that the model satisfies NA, and let C be a claim. Then

$$
\Pi(C) \subseteq \Pi_{\mathbb{Z}}(C) \subseteq \operatorname{cl}(\Pi(C)) .
$$

Moreover, if $\sup \Pi_{\mathbb{Z}}(C) \in \Pi_{\mathbb{Z}}(C)$, then either $C$ has a replication strategy in $\mathcal{Q}$ or there is no cheapest classical superhedging strategy that is in $\mathcal{Q}$.

Proof The first inclusion is trivial. Proposition 2.12 in combination with NA yields that $\mathfrak{Q}_{\mathbb{Z}}^{\max }$ is the set of martingale measures which are equivalent to $P$ and that this set is dense in $\mathfrak{Q}_{\mathbb{Z}}$. Thus Proposition 3.4 implies

$$
\begin{aligned}
\Pi_{\mathbb{Z}}(C) & \subseteq\left\{E_{Q}\left[\frac{C}{(1+r)^{T}}\right]: Q \in \mathfrak{Q}_{\mathbb{Z}}\right\} \\
& \subseteq \operatorname{cl}\left(\left\{E_{Q}\left[\frac{C}{(1+r)^{T}}\right]: Q \in \mathfrak{Q}_{\mathbb{Z}}^{\max }\right\}\right) \\
& =\operatorname{cl}(\Pi(C)) .
\end{aligned}
$$

To show the second assertion, suppose that $s:=\sup \Pi_{\mathbb{Z}}(C) \in \Pi_{\mathbb{Z}}(C)$ and that there is a cheapest classical superhedging strategy $\bar{\varphi} \in \mathcal{Q}$. This means that $\bar{\varphi}$ has price $V_{0}(\bar{\varphi})=s$ and payoff $V_{T}(\bar{\varphi}) \geq C$. Since $s \in \Pi_{\mathbb{Z}}(C)$, there is an integerarbitrage-free extension of the model where $C$ trades at price $s$. Consider the strategy $(\bar{\varphi},-1)$ in the extended model. Its cost is zero, and its payoff is $V_{T}(\bar{\varphi})-C \geq 0$. By 
part (i) of Lemma 2.5, we conclude that $C=V_{T}(\bar{\varphi})$, and so $\bar{\varphi} \in \mathcal{Q}$ is a replication strategy for $C$.

An alternative proof of the inclusion $\Pi_{\mathbb{Z}}(C) \subseteq \operatorname{cl}(\Pi(C))$ can be done using Lemma 2.5(i), Lemma 2.6(i) and Theorem 3.2.

In the preceding theorem, the interval boundaries may or may not be contained in $\Pi_{\mathbb{Z}}(C)$, as the following example shows. The computations needed for parts (ii)-(iv) are similar to (i), and we omit the details.

Example 3.7 Let $\Omega=\left\{\omega_{1}, \omega_{2}, \omega_{3}\right\}, r=0, T=1$ and assume that the number of risky assets is $d=1$. Let $S_{0}^{1}=2$ and

$$
S_{1}^{1}\left(\omega_{j}\right)= \begin{cases}1, & j=1, \\ 3, & j=2, \\ 3, & j=3 .\end{cases}
$$

The equivalent martingale measures are given by

$$
Q_{\alpha}:=\frac{1}{2} \delta_{\omega_{1}}+\alpha \delta_{\omega_{2}}+\left(\frac{1}{2}-\alpha\right) \delta_{\omega_{3}}, \quad \alpha \in\left(0, \frac{1}{2}\right),
$$

and so the model satisfies NA.

(i) Define the claim

$$
C\left(\omega_{j}\right)= \begin{cases}2 \sqrt{2}, & j=1, \\ 0, & j=2, \\ 4 \sqrt{2}, & j=3 .\end{cases}
$$

Using (3.5), we find the classical arbitrage-free prices

$$
\Pi(C)=\left\{E_{Q_{\alpha}}[C]: \alpha \in\left(0, \frac{1}{2}\right)\right\}=(\sqrt{2}, 3 \sqrt{2}) .
$$

We now check the boundary points for integer arbitrage, using part (iii) of Lemma 2.5. An integer arbitrage in the market extended by $C$ with price $p$ thus amounts to finding a vector $\varphi \in \mathbb{Z}^{2}$ such that $\varphi\left(\Delta S_{1}^{1}, C-p\right) \geq 0$ and $\varphi\left(\Delta S_{1}^{1}, C-p\right) \neq 0$. For $p=\sqrt{2}$, we get the inequalities

$$
\left(\begin{array}{cc}
-1 & \sqrt{2} \\
1 & -\sqrt{2} \\
1 & 3 \sqrt{2}
\end{array}\right)\left(\begin{array}{l}
\varphi^{1} \\
\varphi^{2}
\end{array}\right) \geq 0
$$

The solution set

$$
\left\{\left(\varphi^{1}, \varphi^{1} / \sqrt{2}\right): \varphi^{1} \in[0, \infty)\right\}
$$

has trivial intersection with $\mathbb{Z}^{2}$, and so $\sqrt{2}$ is an integer-arbitrage-free price for $C$. Similarly, we obtain that $3 \sqrt{2} \in \Pi_{\mathbb{Z}}(C)$ as well, and we conclude that the interval $\Pi_{\mathbb{Z}}(C)$ contains both endpoints, i.e., $\Pi_{\mathbb{Z}}(C)=[\sqrt{2}, 3 \sqrt{2}]$. 
We now verify that there is no cheapest classical superhedge in $\mathcal{Q}$, in accordance with the second assertion of Theorem 3.6. (Note that $C$ is not replicable as $|\Pi(C)|>1$; in particular, there is no replication strategy in $\mathcal{Q}$.) Clearly, if $\bar{\varphi} \in \mathbb{R}^{2}$ is a cheapest superhedge, then $\varphi^{0}$ must satisfy $\varphi^{0}=\max _{\omega \in \Omega}\left(C(\omega)-\varphi^{1} S_{1}^{1}(\omega)\right)$. The cost of this strategy is then

$$
V_{0}(\bar{\varphi})=\max _{\omega \in \Omega}\left(C(\omega)-\varphi^{1} S_{1}^{1}(\omega)\right)+\varphi^{1} S_{0}^{1}=\max _{\omega \in \Omega}\left(C(\omega)-\varphi^{1} \Delta S_{1}^{1}(\omega)\right) .
$$

Our optimal strategy is $\bar{\varphi}=(3 \sqrt{2}, \sqrt{2}) \notin \mathcal{Q}$, because the problem

$$
\inf _{\varphi^{1} \in \mathbb{R}} \max _{\omega \in \Omega}\left(C(\omega)-\varphi^{1} \Delta S_{1}^{1}(\omega)\right)=\inf _{\varphi^{1} \in \mathbb{R}} \max \left(2 \sqrt{2}+\varphi^{1},-\varphi^{1}, 4 \sqrt{2}-\varphi^{1}\right)
$$

has the unique solution $\varphi^{1}=\sqrt{2}$. Similarly, we obtain that the most expensive classical subhedge is not in $\mathcal{Q}$, agreeing with the (obvious) subhedging variant of the second assertion of Theorem 3.6.

(ii) If

$$
C\left(\omega_{j}\right)= \begin{cases}2 \sqrt{2}, & j=1, \\ 0, & j=2, \\ 2 \sqrt{2}, & j=3,\end{cases}
$$

then $\Pi_{\mathbb{Z}}(C)=[\sqrt{2}, 2 \sqrt{2})$. The cheapest classical superhedge is in $\mathcal{Q}$, while the most expensive classical subhedge is not in $\mathcal{Q}$.

(iii) If

$$
C\left(\omega_{j}\right)= \begin{cases}0, & j=1, \\ 0, & j=2, \\ 2 \sqrt{2}, & j=3,\end{cases}
$$

then $\Pi_{\mathbb{Z}}(C)=(0, \sqrt{2}]$. The cheapest classical superhedge is not in $\mathcal{Q}$, whereas the most expensive classical subhedge is in $\mathcal{Q}$.

(iv) If

$$
C\left(\omega_{j}\right)= \begin{cases}0, & j=1, \\ 0, & j=2, \\ 2, & j=3,\end{cases}
$$

then $\Pi_{\mathbb{Z}}(C)=(0,1)$. The cheapest classical superhedge and the most expensive classical subhedge are both in $\mathcal{Q}$.

It might make sense to restrict attention to static trading strategies in the claim, e.g. as a simple approach for modelling the typically reduced liquidity of derivatives compared to their underlyings. This means that the claim can initially be bought or sold, but then not traded until maturity. In the classical case, the superhedging theorem (Theorem 3.2) readily yields that the set $\Pi^{\text {stat }}(C)$ of static-arbitrage-free claim prices defined in this way satisfies

$$
\Pi^{\text {stat }}(C)=\Pi(C) .
$$


Now suppose that our model satisfies NIA, but not necessarily NA. Analogously to $(3.1)$, define

$$
\hat{\sigma}_{\mathbb{Z}}(C)=\sup \left\{V_{0}(\bar{\varphi}): \bar{\varphi} \in \mathcal{Z}, V_{T}(\bar{\varphi}) \leq C\right\} .
$$

For $p \notin\left[\hat{\sigma}_{\mathbb{Z}}(C), \sigma_{\mathbb{Z}}(C)\right]$, we clearly have $p \notin \Pi_{\mathbb{Z}}^{\text {stat }}(C)$ because by using appropriate integer sub- resp. superhedges, one can easily construct a static integer arbitrage for the extended model. Therefore, we obtain

$$
\Pi_{\mathbb{Z}}(C) \subseteq \Pi_{\mathbb{Z}}^{\text {stat }}(C) \subseteq\left[\hat{\sigma}_{\mathbb{Z}}(C), \sigma_{\mathbb{Z}}(C)\right]
$$

We now proceed to identify the value of the 'cheapest' superhedge in $\mathcal{Q}$. The only difference to the classical case is that the cheapest superhedge is not necessarily in $\mathcal{Q}$, but can be approximated arbitrarily well with superhedges in $\mathcal{Q}$ (even if only NIA holds). For results on the 'cheapest' superhedge in $\mathcal{Z}$, see Sect. 5.

Proposition 3.8 Under Assumption 2.1, suppose that the model satisfies NIA and let $C$ be a claim. Then there is a cheapest superhedge $\bar{\varphi} \in \mathcal{R}$ which in addition satisfies

$$
V_{0}(\bar{\varphi})=\sup \left\{E_{Q}\left[\frac{C}{(1+r)^{T}}\right]: Q \in \mathfrak{Q}_{\mathbb{Z}}\right\}=\sup \left\{E_{Q}\left[\frac{C}{(1+r)^{T}}\right]: Q \in \mathfrak{Q}_{\mathbb{Z}}^{\max }\right\} .
$$

Moreover, for any $\epsilon>0$, there is a superhedge $\bar{\xi} \in \mathcal{Q}$ with $V_{0}(\bar{\xi}) \leq V_{0}(\bar{\varphi})+\epsilon$.

Proof Proposition 2.12 yields

$$
\sup \left\{E_{Q}\left[\frac{C}{(1+r)^{T}}\right]: Q \in \mathfrak{Q}_{\mathbb{Z}}\right\}=\sup \left\{E_{Q}\left[\frac{C}{(1+r)^{T}}\right]: Q \in \mathfrak{Q}_{\mathbb{Z}}^{\max }\right\} .
$$

Let $Q \in \mathfrak{Q}_{\mathbb{Z}}^{\max }$ and define

$$
A:=\{\omega \in \Omega: Q[\{\omega\}]=0\} .
$$

Then $\bar{S}$ restricted to $\Omega \backslash A$ satisfies NA because $Q$ is a martingale measure. By Assumption 2.1, there is a cheapest superhedge for $C$ on this market, which we denote by $\bar{\eta} \in \mathcal{R}$. It satisfies $V_{T}(\bar{\eta}) \geq C Q$-a.s. Since $Q \in \mathfrak{Q}_{\mathbb{Z}}^{\max }$, there is $\bar{\psi} \in \mathcal{R}_{0}$ such that $V_{T}(\bar{\psi}) \geq 0$ and $\left\{V_{T}(\bar{\psi})>0\right\}=A$. As $\Omega$ is finite, there is $a \in \mathbb{R}$ such that

$$
V_{T}(a \bar{\psi}+\bar{\eta})=a V_{T}(\bar{\psi})+V_{T}(\bar{\eta}) \geq C \quad \text { pointwise. }
$$

By Proposition 2.12 and Theorem 3.2, we find that $\bar{\varphi}:=a \bar{\psi}+\bar{\eta}$ is a superhedge for $C$ with initial price

$$
V_{0}(\bar{\varphi})=V_{0}(\bar{\eta})=\sup \left\{E_{Q}\left[\frac{C}{(1+r)^{T}}\right]: Q \in \mathfrak{Q}_{\mathbb{Z}}^{\max }\right\} .
$$

Now let $\bar{\gamma} \in \mathcal{R}$ be any superhedge for $C$. Then $V_{T}(\bar{\gamma}) \geq C Q$-a.s. for any $Q \in \mathfrak{Q}_{\mathbb{Z}}^{\max }$, and hence 


$$
V_{0}(\bar{\gamma})=E_{Q}\left[\frac{V_{T}(\bar{\gamma})}{(1+r)^{T}}\right] \geq \sup \left\{E_{Q}\left[\frac{C}{(1+r)^{T}}\right]: Q \in \mathfrak{Q}_{\mathbb{Z}}^{\max }\right\} .
$$

Consequently, $\bar{\varphi}$ is a cheapest superhedge for $C$.

The second assertion follows easily from part (i) of Lemma 2.6.

\section{The structure of the set of integer-arbitrage-free prices}

The main result of this section is that the set $\Pi_{\mathbb{Z}}(C)$ of NIA-compatible claim prices is always dense in an interval (Theorem 4.3). First, we give some sufficient conditions that imply that $\Pi_{\mathbb{Z}}(C)$ equals an interval.

Proposition 4.1 Let Assumption 2.1 hold and assume that the model satisfies NIA. Then $\Pi_{\mathbb{Z}}(C)$ is an interval if any of the following statements holds:

(i) There is only one trading period $(T=1)$, and $\Pi_{\mathbb{Z}}(C)$ is not empty.

(ii) There is only one risky asset $(d=1)$.

(iii) The model satisfies NA.

Proof If (ii) holds, then Theorem 2.7 yields that (iii) holds. If we assume (iii), then Theorem 3.6 yields the claim. Now assume that (i) holds. Proposition 3.4 implies that

$$
\Pi_{\mathbb{Z}}(C) \subseteq\left\{E_{Q}\left[\frac{C}{(1+r)^{T}}\right]: Q \in \mathfrak{Q}_{\mathbb{Z}}\right\}=: J
$$

If $J$ is a singleton, we have equality by the assumption $\Pi_{\mathbb{Z}}(C) \neq \emptyset$, and the claim follows. Thus we may assume that $J$ contains at least two points. The set $J$ is an interval because $\mathfrak{Q}_{\mathbb{Z}}$ is convex. Let $p \in \operatorname{int}(J)$ and define $X_{0}:=p, X_{1}:=C$. Assume for a contradiction that there is an integer arbitrage $\left(\bar{\varphi}, \varphi^{d+1}\right)$ for the model $\left(S^{0}, \ldots, S^{d}, X\right)$. By part (ii) of Lemma 2.5 , we may assume that $V_{0}\left(\bar{\varphi}, \varphi^{d+1}\right)=0$. We have $\varphi_{1}^{d+1} \neq 0$, because otherwise $\bar{\varphi}$ is an integer arbitrage for $\left(S^{0}, \ldots, S^{d}\right)$ with $V_{0}(\bar{\varphi})=0$.

Case 1: $\varphi_{1}^{d+1}<0$. Then $C \leq-\left(1 / \varphi_{1}^{d+1}\right) \sum_{j=0}^{d} \varphi_{1}^{j} S_{1}^{j}$. Thus,

$$
\psi_{1}^{j}:=-\frac{\varphi^{j}}{\varphi^{d+1}}, \quad j=0, \ldots, d,
$$

is a superhedge for $C$. We have $V_{0}(\bar{\psi})=p$, and Proposition 3.8 yields that we have $p=V_{0}(\bar{\psi}) \geq \sup (J)>p$, which is a contradiction.

Case 2: $\varphi_{1}^{d+1}>0$. Analogous.

Thus $p \in \Pi_{\mathbb{Z}}(C)$, which yields that $\operatorname{int}(J) \subseteq \Pi_{\mathbb{Z}}(C) \subseteq J$, and hence $\Pi_{\mathbb{Z}}(C)$ is an interval.

We now give an example in which the set of no-integer-arbitrage-compatible prices is not an interval. More precisely, we exhibit a model satisfying NIA and a claim $C$ whose set of NIA-consistent prices is given by

$$
\Pi_{\mathbb{Z}}(C)=[0,1 / 2] \backslash(\mathbb{Q}+\mathbb{Q} \pi) .
$$


Example 4.2 Let $\Omega:=\left\{\omega_{1}, \omega_{2}, \omega_{3}, \omega_{4}\right\}, d=2, T=2$ and $r=0$. We use the filtration $\mathcal{F}_{0}:=\{\emptyset, \Omega\}, \mathcal{F}_{1}:=\sigma\left(\left\{\omega_{1}\right\},\left\{\omega_{2}, \omega_{3}\right\},\left\{\omega_{4}\right\}\right)$ and $\mathcal{F}_{2}:=2^{\Omega}$. The market model is given by $\left(S_{0}^{1}, S_{0}^{2}\right):=(1, \pi)$ and

$$
S_{2}\left(\omega_{j}\right):=S_{1}\left(\omega_{j}\right):= \begin{cases}(3 / 2,3 \pi / 2), & j=1, \\ (1 / 2, \pi / 2), & j=2,3, \\ (1,1+\pi), & j=4 .\end{cases}
$$

This market allows the static real arbitrage $\bar{\eta}_{t}:=(0,-\pi, 1)$, which is self-financing and satisfies $V_{0}(\bar{\eta})=0$ and $V_{2}(\bar{\eta})=1_{\left\{\omega_{4}\right\}}$. Thus any martingale measure $Q$ must satisfy $Q\left[\left\{\omega_{4}\right\}\right]=0$. For any $\alpha \in[0,1]$, define the measure $Q_{\alpha}$ by

$$
Q_{\alpha}\left[\left\{\omega_{j}\right\}\right]:= \begin{cases}1 / 2, & j=1, \\ \alpha / 2, & j=2, \\ (1-\alpha) / 2, & j=3, \\ 0, & j=4 .\end{cases}
$$

Then $Q_{\alpha}$ is a martingale measure and $\mathcal{Z}_{Q_{\alpha}}^{0}=\{0\}$. In particular, Theorem 2.13 yields that NIA holds. Moreover, $\mathfrak{Q}_{\mathbb{Z}}=\left\{Q_{\alpha}: \alpha \in[0,1]\right\}$.

Now we choose the claim $C:=1_{\left\{\omega_{2}\right\}}$. Proposition 3.4 yields

$$
\Pi_{\mathbb{Z}}(C) \subseteq\left\{E_{Q}[C]: Q \in \mathfrak{Q}_{\mathbb{Z}}\right\}=[0,1 / 2] .
$$

Let $p \in[0,1 / 2] \cap(\mathbb{Q}+\mathbb{Q} \pi), p \neq 0$, and assume for a contradiction that $p \in \Pi_{\mathbb{Z}}(C)$. Then there is an adapted process $\left(X_{t}\right)_{t=0,1,2}$ such that $X_{0}=p, X_{2}=C$ and the model $\left(S^{0}, S^{1}, S^{2}, X\right)$ satisfies NIA. We have

$$
\hat{\mathfrak{Q}}_{\mathbb{Z}} \subseteq \mathfrak{Q}_{\mathbb{Z}}=\left\{Q_{\alpha}: \alpha \in[0,1]\right\},
$$

where $\hat{\mathfrak{Q}}_{\mathbb{Z}}$ denotes the analogue of $\mathfrak{Q}_{\mathbb{Z}}$ for the extended model. Hence by Theorem 2.13 (our FTAP), there is some $Q_{\alpha}$ satisfying $E_{Q_{\alpha}}[C]=p$. Clearly, this holds only for $Q_{2 p}$, and we obtain

$$
X_{1}=E_{Q_{2 p}}\left[C \mid \mathcal{F}_{1}\right]=2 p 1_{\left\{\omega_{2}, \omega_{3}\right\}} .
$$

Now let $u, v \in \mathbb{Q}$ be such that $2 p=u+v \pi$. Define the strategy $\left(\bar{\varphi}_{t}, \varphi_{t}^{3}\right)_{t=1,2} \in \mathcal{Q}$ by

$$
\varphi_{1}^{3}:=\operatorname{sgn}((2-\pi) v-u) \in\{-1,1\}
$$

and

$$
\begin{aligned}
\left(\bar{\varphi}_{1}, \varphi_{1}^{3}\right) & :=\left(-3 p \varphi_{1}^{3}, u \varphi_{1}^{3}, v \varphi_{1}^{3}, \varphi_{1}^{3}\right), \\
\left(\bar{\varphi}_{2}, \varphi_{2}^{3}\right)\left(\omega_{j}\right) & := \begin{cases}0, & j=1,2,3, \\
\left(\frac{1}{2}|(2-\pi) v-u|, 0,0,0\right), & j=4 .\end{cases}
\end{aligned}
$$


This strategy satisfies $V_{0}\left(\bar{\varphi}, \varphi^{3}\right)=0$. At time 1 , we have

$$
V_{1}\left(\bar{\varphi}, \varphi^{3}\right)=\varphi_{1}^{3}\left(-3 p+u S_{1}^{1}+v S_{1}^{2}+2 p 1_{\left\{\omega_{2}, \omega_{3}\right\}}\right) .
$$

This vanishes on $\left\{\omega_{1}, \omega_{2}, \omega_{3}\right\}$, and so

$$
\begin{aligned}
V_{1}\left(\bar{\varphi}, \varphi^{3}\right) & =\varphi_{1}^{3}(-3 p+u+(1+\pi) v) 1_{\left\{\omega_{4}\right\}} \\
& =\frac{1}{2} \varphi_{1}^{3}((2-\pi) v-u) 1_{\left\{\omega_{4}\right\}} \\
& =\frac{1}{2}|(2-\pi) v-u| 1_{\left\{\omega_{4}\right\}} .
\end{aligned}
$$

We see that $\left(\bar{\varphi}, \varphi^{3}\right)$ is self-financing, and since

$$
V_{2}\left(\bar{\varphi}, \varphi^{3}\right)=V_{1}\left(\bar{\varphi}, \varphi^{3}\right)=\frac{1}{2}|(2-\pi) v-u| 1_{\left\{\omega_{4}\right\}},
$$

it is a rational arbitrage. This gives a contradiction.

Now let $p=0$ (which was excluded in the previous step) and assume for a contradiction that $p \in \Pi_{\mathbb{Z}}(C)$. Then there is an adapted process $\left(X_{t}\right)_{t=0,1,2}$ such that $X_{0}=0, X_{2}=C$ and the model $\left(S^{0}, S^{1}, S^{2}, X\right)$ satisfies NIA. Define the static strategy $\left(\bar{\varphi}, \varphi^{3}\right):=(0,0,0,1)$. We have $V_{0}\left(\bar{\varphi}, \varphi^{3}\right)=0$ and $V_{2}\left(\bar{\varphi}, \varphi^{3}\right)=1_{\left\{\omega_{2}\right\}}$. Thus $\left(\bar{\varphi}, \varphi^{3}\right)$ is an integer arbitrage, which is again a contradiction.

We have shown so far that $\Pi_{\mathbb{Z}}(C) \subseteq[0,1 / 2] \backslash(\mathbb{Q}+\mathbb{Q} \pi)$. Conversely, let now $p \in[0,1 / 2] \backslash(\mathbb{Q}+\mathbb{Q} \pi)$. We show that $p \in \Pi_{\mathbb{Z}}(C)$. Define $\alpha:=2 p$ and $X_{0}^{\alpha}:=\alpha / 2$, $X_{1}^{\alpha}:=\alpha 1_{\left\{\omega_{2}, \omega_{3}\right\}}, X_{2}^{\alpha}:=C$. To see that the model $\left(S^{0}, S^{1}, S^{2}, X^{\alpha}\right)$ satisfies NIA, assume for a contradiction that there is an integer arbitrage. Then there is a one-period arbitrage $\left(\bar{\varphi}, \varphi^{3}\right)$. Obviously, there is no arbitrage possibility in the second period, and so we may assume $\left(\varphi_{2}, \varphi_{2}^{3}\right)=0$ (i.e., no risky position in the second period). Thus $V_{1}\left(\bar{\varphi}, \varphi^{3}\right)=V_{2}\left(\bar{\varphi}, \varphi^{3}\right) \geq 0$. Since $Q_{\alpha}$ is a martingale measure for the extended model, we get $V_{1}\left(\bar{\varphi}, \varphi^{3}\right)=0 Q_{\alpha}$-a.s. In particular, $V_{1}\left(\bar{\varphi}, \varphi^{3}\right)\left(\omega_{2}\right)=0$, and together with $V_{0}\left(\bar{\varphi}, \varphi^{3}\right)=0($ see Lemma $2.5($ ii) $)$, this implies

$$
\varphi_{1}^{1}+\pi \varphi_{1}^{2}-\alpha \varphi_{1}^{3}=0
$$

As the original model satisfies NIA, we must have $\varphi_{1}^{3} \neq 0$, which leads to the contradiction

$$
2 p=\alpha=\frac{\varphi_{1}^{1}+\pi \varphi_{1}^{2}}{\varphi_{1}^{3}} \in \mathbb{Q}+\mathbb{Q} \pi .
$$

Thus, there is no integer arbitrage, i.e., the model satisfies NIA, and hence $p \in \Pi_{\mathbb{Z}}(C)$.

Throughout the remainder of this section, we suppose that Assumption 2.1 and NIA hold. Also, let $C$ be a claim. The following theorem is our main result on the structure of $\Pi_{\mathbb{Z}}(C)$ in the general case. 
Theorem 4.3 The set $\Pi_{\mathbb{Z}}(C)$ is either empty or dense in

$$
\left[\inf \left\{E_{Q}\left[\frac{C}{(1+r)^{T}}\right]: Q \in \mathfrak{Q}_{\mathbb{Z}}^{\max }\right\}, \sup \left\{E_{Q}\left[\frac{C}{(1+r)^{T}}\right]: Q \in \mathfrak{Q}_{\mathbb{Z}}^{\max }\right\}\right] .
$$

The theorem will follow from Lemmas 4.10 and 4.11 below. Note that $\mathfrak{Q}_{\mathbb{Z}}^{\max }$ can be replaced by $\mathfrak{Q}_{\mathbb{Z}}$, due to the last assertion of Proposition 2.12. In order to prove Theorem 4.3, we assume that $\Pi_{\mathbb{Z}}(C)$ is nonempty and choose $p^{*} \in \Pi_{\mathbb{Z}}(C)$. By definition, there is an adapted process $\left(X_{t}^{*}\right)_{t \in \mathbb{T}}$ such that $X_{0}^{*}=p, X_{T}^{*}=C$ and the model $\left(S^{0}, \ldots, S^{d}, X^{*}\right)$ satisfies NIA. We also define

$$
A_{t}:=\left\{\omega \in \Omega: \exists \bar{\varphi} \in \mathcal{R}_{0} \text { with } V_{T}(\bar{\varphi}) \geq 0, V_{T}(\bar{\varphi})(\omega)>0, \bar{\varphi}_{1}, \ldots, \bar{\varphi}_{t}=0\right\}
$$

for any $t \in \mathbb{T}$. By the same argument as in the proof of Theorem 2.13, there is $\bar{\varphi} \in \mathcal{R}_{0}$ with $\bar{\varphi}_{1}, \ldots, \bar{\varphi}_{t}=0, V_{T}(\bar{\varphi}) \geq 0$ and $\left\{V_{T}(\bar{\varphi})>0\right\}=A_{t}$. From the definition, we see that $A_{t} \subseteq A_{t-1}$. We first establish the following measurability statement.

Lemma 4.4 For any $t \in\{1, \ldots, T\}$, the set $A_{t-1} \backslash A_{t}$ is in $\mathcal{F}_{t}$.

Proof We denote by $\mathcal{R}_{0}^{1}$ the set of one-step strategies, i.e., the set of strategies $\varphi \in \mathcal{R}_{0}$ such that there is $t_{0} \in \mathbb{T} \backslash\{0\}$ with $\varphi_{t}=0$ for any $t \in \mathbb{T} \backslash\left\{t_{0}\right\}$. Proposition 5.11 in [13] (asserting the equivalence of arbitrage to one-period arbitrage) yields

$$
A_{t}=\left\{\omega \in \Omega: \exists \bar{\varphi} \in \mathcal{R}_{0}^{1} \text { with } V_{T}(\bar{\varphi}) \geq 0, V_{T}(\bar{\varphi})(\omega)>0, \bar{\varphi}_{1}, \ldots, \bar{\varphi}_{t}=0\right\} .
$$

Thus we get

$$
\begin{aligned}
& A_{t-1} \backslash A_{t}=\left\{\omega \in \Omega: \exists \bar{\varphi} \in \mathcal{R}_{0}^{1}\right. \text { with } \\
& \left.\qquad V_{T}(\bar{\varphi}) \geq 0, V_{T}(\bar{\varphi})(\omega)>0, \bar{\varphi}_{1}, \ldots, \bar{\varphi}_{t-1}=0, \varphi_{t} \neq 0\right\} \\
& =\bigcup_{\left\{\bar{\varphi} \in \mathcal{R}_{0}^{1}: V_{T}(\bar{\varphi}) \geq 0, \bar{\varphi}_{1}, \ldots, \bar{\varphi}_{t-1}=0, \varphi_{t} \neq 0\right\}}\left\{\omega \in \Omega: V_{T}(\bar{\varphi})(\omega)>0\right\} .
\end{aligned}
$$

Let $\bar{\varphi} \in \mathcal{R}_{0}^{1}$ be such that $V_{T}(\bar{\varphi}) \geq 0, \bar{\varphi}_{1}, \ldots, \bar{\varphi}_{t-1}=0, \varphi_{t} \neq 0$. Then we have $\varphi_{s}=0$ for any $s=t+1, \ldots, T$, and hence $V_{t}(\bar{\varphi})=\frac{V_{T}(\bar{\varphi})}{(1+r)^{T-t}}$. This implies that

$$
\left\{\omega \in \Omega: V_{T}(\bar{\varphi})(\omega)>0\right\}=\left\{\omega \in \Omega: V_{t}(\bar{\varphi})(\omega)>0\right\} \in \mathcal{F}_{t} .
$$

Hence $A_{t-1} \backslash A_{t} \in \mathcal{F}_{t}$ because in a finite $\sigma$-algebra, any union of measurable sets is measurable.

In Definition 4.6 below, we need measures with a certain non-degeneracy condition, namely that any nonempty $\mathcal{F}_{t}$-measurable set has positive mass, while the measure is supported on $\Omega \backslash A_{t}$. We first characterise this property.

Lemma 4.5 Let $t \in \mathbb{T}$ and $Q$ be a probability measure on $(\Omega, \mathcal{A})$ which is supported on $\Omega \backslash A_{t}$. Then the following statements are equivalent: 
(1) For any $B \in \mathcal{F}_{t}$ with $B \neq \emptyset$, we have $Q[B]>0$.

(2) For any $B \in \mathcal{F}_{t}$ with $B \cap\left(\Omega \backslash A_{t}\right) \neq \emptyset$, we have $Q[B]>0$.

Proof The implication (1) $\Rightarrow$ (2) is trivial. We assume (2). Let $B \in \mathcal{F}_{t}$ be nonempty. Assume for a contradiction that $B \subseteq A_{t}$. Let $\bar{\varphi} \in \mathcal{R}_{0}$ be an arbitrage with $\bar{\varphi}_{0}=\cdots=\bar{\varphi}_{t}=0$ and $\left\{V_{T}(\varphi)>0\right\}=A_{t}$. Let $\left(\bar{\psi}^{(N)}\right)_{N \in \mathbb{N}}$ be a sequence in $\mathcal{Q}_{0}$ such that $\bar{\psi}^{(N)} \rightarrow \bar{\varphi}$ pointwise for $N \rightarrow \infty$ and $\bar{\psi}_{0}^{(N)}=\cdots=\bar{\psi}_{t}^{(N)}=0$. Then $\bar{\psi}^{(N)} 1_{B}$ is in $\mathcal{Q}_{0}$ and converges to $\bar{\varphi} 1_{B}$ for $N \rightarrow \infty$. Consequently, there is $N \in \mathbb{N}$ such that $\left\{V_{T}\left(\bar{\psi}^{(N)} 1_{B}\right)>0\right\}=B$. Hence $\bar{\psi}^{(N)} 1_{B}$ is a rational arbitrage, which contradicts (NIA) (which we assumed for this section before Theorem 4.3).

Consequently, we have $B \nsubseteq A_{t}$. In particular, we have $B \cap\left(\Omega \backslash A_{t}\right) \neq \emptyset$. Since we assumed (2), we get $Q[B]>0$ as required.

Definition 4.6 We write $\mathfrak{Q}_{t}$ for the set of measures $Q$ such that $\left(\hat{S}_{u}\right)_{u=t, \ldots, T}$ is a $Q$-martingale, $\Omega \backslash A_{t}$ is the support of $Q$, and $Q[B]>0$ for any nonempty set $B \in \mathcal{F}_{t}$. Now we define two sequences of sets via

$$
\begin{aligned}
& \mathcal{K}_{T}:=\{C\}, \\
& \mathcal{K}_{t}:=\left\{E_{Q}\left[\frac{D}{1+r} \mid \mathcal{F}_{t}\right]: Q \in \mathfrak{Q}_{t}, D \in \mathcal{K}_{t+1}\right\}, \\
& \mathcal{C}_{T}:=\{C\}, \\
& \mathcal{C}_{t}:=\left\{E_{Q}\left[\frac{D}{1+r} \mid \mathcal{F}_{t}\right]: Q \in \mathfrak{Q}_{t}, D \in \mathcal{C}_{t+1}, \forall B \in \mathcal{F}_{t}, \forall \xi \in \mathbb{Q}^{d}, \forall s \in\{-1,1\}:\right. \\
& 1_{B} \xi \Delta \hat{S}_{t+1} \geq s 1_{B}\left(\frac{D}{(1+r)^{t+1}}-E_{Q}\left[\frac{D}{(1+r)^{t+1}} \mid \mathcal{F}_{t}\right]\right) \\
&\left.\Rightarrow 1_{B} \xi \Delta \hat{S}_{t+1}=s 1_{B}\left(\frac{D}{(1+r)^{t+1}}-E_{Q}\left[\frac{D}{(1+r)^{t+1}} \mid \mathcal{F}_{t}\right]\right)\right\}
\end{aligned}
$$

for any $t \in \mathbb{T} \backslash\{T\}$.

Lemma 4.8 below, together with the convexity of $\mathfrak{Q}_{\mathbb{Z}}^{\max }=\mathfrak{Q}_{0}$, implies that

$$
\mathcal{K}_{0}=\left[\inf \left\{E_{Q}\left[\frac{C}{(1+r)^{T}}\right]: Q \in \mathfrak{Q}_{\mathbb{Z}}^{\max }\right\},\left\{E_{Q}\left[\frac{C}{(1+r)^{T}}\right]: Q \in \mathfrak{Q}_{\mathbb{Z}}^{\max }\right\}\right],
$$

and Lemma 4.11 below yields a countable exception set $F$ with the property that $\mathcal{C}_{0}=\mathcal{K}_{0} \backslash F$. Finally, Lemma 4.10 states that $\mathcal{C}_{0}$ is contained in the set $\Pi_{\mathbb{Z}}(C)$ of NIAcompatible prices, which establishes Theorem 4.3. For technical reasons, we first analyse the sets $\mathfrak{Q}_{t}$, and we need the stochastic convexity of $\mathcal{K}_{t}$ given in Lemma 4.9 below. 
Lemma 4.7 Let $t \in \mathbb{T}$. Then $\mathfrak{Q}_{t}$ is nonempty. If $t \neq 0$, then for any $Q \in \mathfrak{Q}_{t-1}$, there is $Q^{\prime} \in \mathfrak{Q}_{t}$ such that

$$
E_{Q^{\prime}}\left[X \mid \mathcal{F}_{s}\right]=E_{Q}\left[X \mid \mathcal{F}_{s}\right] \quad Q \text {-a.s. }
$$

for any $s=t, \ldots, T$ and any random variable $X: \Omega \rightarrow \mathbb{R}$.

Proof Let $I:=\{t \in \mathbb{T}:$ the claim holds for $t\}$. We have $0 \in I$ by Theorem 2.13(i). Let $t \in \mathbb{T}$ be such that $t-1 \in I$. We show $t \in I$ which implies $I=\mathbb{T}$, and hence the claim. We directly produce the measure with the given extra property. To this end, let $Q \in \mathfrak{Q}_{t-1}$. Let $B_{1}, \ldots, B_{m}$ be an enumeration of the minimal nonempty elements of $\mathcal{F}_{t}$ and define

$$
k:=\left|\left\{B_{\ell}: \ell=1, \ldots, m, B_{\ell} \subseteq A_{t-1} \backslash A_{t}\right\}\right| .
$$

We may assume that $B_{1}, \ldots, B_{k} \subseteq A_{t-1} \backslash A_{t}$. Since $\Omega \backslash A_{t-1}$ is the support of $Q$, we have $Q\left[B_{\ell}\right]>0$ for any $\ell=k+1, \ldots, m$. As $A_{t} \backslash A_{t-1}$ is in $\mathcal{F}_{t}$ by Lemma 4.4, we have $A_{t-1} \backslash A_{t}=\bigcup_{\ell=1}^{k} B_{\ell}$. Define the probability measures $P_{\ell}:=\frac{P}{P\left[B_{\ell}\right]}$ on $B_{\ell}$. Since the model $\left(\hat{S}_{u}\right)_{u=t, \ldots, T}$ satisfies NIA, we get from Theorem 2.13(i) a martingale measure $Q_{\ell} \ll P_{\ell}$ on $B_{\ell}$. Define the probability measure

$$
Q^{\prime}[D]:=\left(Q[D]+\sum_{\ell=1}^{k} Q_{\ell}\left[D \cap B_{\ell}\right]\right) /(1+k), \quad D \in \mathcal{A}
$$

Clearly, the support of $Q^{\prime}$ is $\Omega \backslash A_{t}$ and $Q^{\prime}[D]>0$ for any $D \in \mathcal{F}_{t}$. Also, $\left(\hat{S}_{u}\right)_{u=t, \ldots, T}$ is a $Q^{\prime}$-martingale. Thus we have $Q^{\prime} \in \mathfrak{Q}_{t}$.

Now let $X: \Omega \rightarrow \mathbb{R}$ be a random variable and $s \in\{t, \ldots, T\}$. We show that $E_{Q^{\prime}}\left[X \mid \mathcal{F}_{S}\right]$ is a version of the $\mathcal{F}_{s}$-conditional expectation of $X$ under $Q$. To this end, let $D \in \mathcal{F}_{s}$ and define $D^{\prime}:=D \backslash A_{t-1}$. Then $D^{\prime}$ is $Q^{\prime}$-essentially $\mathcal{F}_{s}$-measurable, because $A_{t}$ is a $Q^{\prime}$-null set and $A_{t-1} \backslash A_{t}$ is $\mathcal{F}_{t} \subseteq \mathcal{F}_{s}$-measurable. We have

$$
\begin{aligned}
E_{Q}\left[E_{Q^{\prime}}\left[X \mid \mathcal{F}_{S}\right] 1_{D}\right] & =E_{Q}\left[E_{Q^{\prime}}\left[X \mid \mathcal{F}_{S}\right] 1_{D^{\prime}}\right] \\
& =E_{Q}\left[E_{Q^{\prime}}\left[X 1_{D^{\prime}} \mid \mathcal{F}_{S}\right]\right] \\
& =E_{Q}\left[E_{Q}\left[X 1_{D^{\prime}} \mid \mathcal{F}_{S}\right]\right] \\
& =E_{Q}\left[X 1_{D^{\prime}}\right] \\
& =E_{Q}\left[X 1_{D}\right] .
\end{aligned}
$$

Thus $t \in I$.

Lemma 4.8 For any $t \in \mathbb{T}$, we have

$$
\mathcal{K}_{t}=\left\{E_{Q}\left[\frac{C}{(1+r)^{T-t}} \mid \mathcal{F}_{t}\right]: Q \in \mathfrak{Q}_{t}\right\} .
$$


Proof Define

$$
I:=\left\{t \in \mathbb{T}: \mathcal{K}_{t}=\left\{E_{Q}\left[\frac{C}{(1+r)^{T-t}} \mid \mathcal{F}_{t}\right]: Q \in \mathfrak{Q}_{t}\right\}\right\}
$$

Obviously, $T \in I$. Let $t \in I \backslash\{0\}$. We show that $t-1 \in I$, which implies $I=\mathbb{T}$ and hence the claim.

To this end, let $X_{t-1} \in \mathcal{K}_{t-1}$. Then there are $Q \in \mathfrak{Q}_{t-1}$ and $X_{t} \in \mathcal{K}_{t}$ such that $X_{t-1}=E_{Q}\left[\frac{X_{t}}{1+r} \mid \mathcal{F}_{t-1}\right]$. Since $X_{t} \in \mathcal{K}_{t}$, there is $R \in \mathfrak{Q}_{t}$ with $X_{t}=E_{R}\left[\frac{C}{(1+r)^{T-t}} \mid \mathcal{F}_{t}\right]$. Define the measure

$$
Q^{\prime}[B]:=E_{Q}\left[R\left[B \mid \mathcal{F}_{t}\right]\right], \quad B \in \mathcal{A} .
$$

Since $R \in \mathfrak{Q}_{t}$, we have $R[B]>0$ for any nonempty set $B \in \mathcal{F}_{t}$. Let $B \in \mathcal{F}_{t-1} \subseteq \mathcal{F}_{t}$ be nonempty. Then $R\left[B \mid \mathcal{F}_{t}\right]=1_{B}$ and so $Q^{\prime}[B]=Q[B]>0$. Also, $\left(\hat{S}_{u}\right)_{u=t-1, \ldots, T}$ is a $Q^{\prime}$-martingale. Since $A_{t} \subseteq A_{t-1}$ and $A_{t-1} \backslash A_{t} \in \mathcal{F}_{t}$, we get

$$
R\left[A_{t-1} \mid \mathcal{F}_{t}\right]=1_{A_{t-1} \backslash A_{t}}+R\left[A_{t} \mid \mathcal{F}_{t}\right] .
$$

However, $A_{t}$ is an $R$-nullset, so that $R\left[A_{t} \mid \mathcal{F}_{t}\right]=0 R$-a.s. Since $\mathcal{F}_{t}$ has no nonempty $R$-nullsets, we have $R\left[A_{t} \mid \mathcal{F}_{t}\right]=0$. We get $R\left[A_{t-1} \mid \mathcal{F}_{t}\right]=1_{A_{t-1} \backslash A_{t}}$, which yields $Q^{\prime}\left[A_{t-1}\right]=Q\left[A_{t-1} \backslash A_{t}\right]=0$. Let $\omega \in \Omega \backslash A_{t-1}$. Then $R\left[\{\omega\} \mid \mathcal{F}_{t}\right] \geq 0$ and $R\left[\{\omega\} \mid \mathcal{F}_{t}\right](\omega)>0$. Since $Q[\{\omega\}]>0$, we get $Q^{\prime}[\{\omega\}]>0$. Thus the support of $Q^{\prime}$ is $\Omega \backslash A_{t-1}$, which yields $Q^{\prime} \in \mathfrak{Q}_{t-1}$. We have

$$
\begin{aligned}
E_{Q^{\prime}}\left[\frac{C}{(1+r)^{T-(t-1)}} \mid \mathcal{F}_{t-1}\right] & =E_{Q}\left[E_{R}\left[\frac{C}{(1+r)^{T-t}} \mid \mathcal{F}_{t}\right] /(1+r) \mid \mathcal{F}_{t-1}\right] \\
& =E_{Q}\left[\frac{X_{t}}{1+r} \mid \mathcal{F}_{t-1}\right] \\
& =X_{t-1} .
\end{aligned}
$$

Thus

$$
X_{t-1} \in\left\{E_{Q}\left[\frac{C}{(1+r)^{T-t}} \mid \mathcal{F}_{t-1}\right]: Q \in \mathcal{Q}_{t-1}\right\} .
$$

Now let

$$
X_{t-1} \in\left\{E_{Q}\left[\frac{C}{(1+r)^{T-(t-1)}} \mid \mathcal{F}_{t-1}\right]: Q \in \mathfrak{Q}_{t-1}\right\} ;
$$

we have to show that $X_{t-1} \in \mathcal{K}_{t-1}$. There is $Q \in \mathfrak{Q}_{t-1}$ such that

$$
X_{t-1}=E_{Q}\left[\frac{C}{(1+r)^{T-(t-1)}} \mid \mathcal{F}_{t-1}\right] .
$$

By Lemma 4.7, we find $Q^{\prime} \in \mathfrak{Q}_{t}$ such that $E_{Q}\left[Y \mid \mathcal{F}_{S}\right]=E_{Q^{\prime}}\left[Y \mid \mathcal{F}_{S}\right] Q$-a.s. for any random variable $Y: \Omega \rightarrow \mathbb{R}$ and any $s=t, \ldots, T$. Then

$$
X_{t}:=E_{Q^{\prime}}\left[\frac{C}{(1+r)^{T-t}} \mid \mathcal{F}_{t}\right] \in \mathcal{K}_{t}
$$


because $t \in I$. We find

$$
\begin{aligned}
\mathcal{K}_{t-1} & \ni E_{Q}\left[\frac{X_{t}}{1+r} \mid \mathcal{F}_{t-1}\right] \\
& =E_{Q}\left[E_{Q^{\prime}}\left[\frac{C}{(1+r)^{T-t+1}} \mid \mathcal{F}_{t}\right] \mid \mathcal{F}_{t-1}\right] \\
& =E_{Q}\left[\frac{C}{(1+r)^{T-(t-1)}} \mid \mathcal{F}_{t-1}\right] \\
& =X_{t-1} .
\end{aligned}
$$

Thus $t-1 \in I$.

Lemma 4.9 For any $t \in \mathbb{T}$, any $\mathcal{F}_{t}$-measurable random variable $\alpha$ with values in $[0,1]$ and any $X, Y \in \mathcal{K}_{t}$, we have

$$
\alpha X+(1-\alpha) Y \in \mathcal{K}_{t}
$$

Proof Lemma 4.8 yields measures $Q, R \in \mathfrak{Q}_{t}$ such that $X=E_{Q}\left[\frac{C}{(1+r)^{T-t}} \mid \mathcal{F}_{t}\right]$ and $Y=E_{R}\left[\frac{C}{(1+r)^{T-t}} \mid \mathcal{F}_{t}\right]$. Define the measure

$$
Q^{\prime}[B]:=E_{Q}\left[\alpha Q\left[B \mid \mathcal{F}_{t}\right]+(1-\alpha) R\left[B \mid \mathcal{F}_{t}\right]\right] .
$$

It is clear that $Q$ and $Q^{\prime}$ agree on $\mathcal{F}_{t}$, and one easily verifies $Q^{\prime} \in \mathfrak{Q}_{t}$. Let $B \in \mathcal{F}_{t}$. Then

$$
\begin{aligned}
E_{Q^{\prime}}\left[1_{B} \frac{C}{(1+r)^{T-t}}\right]= & E_{Q}\left[\alpha E_{Q}\left[1_{B} \frac{C}{(1+r)^{T-t}} \mid \mathcal{F}_{t}\right]\right. \\
& \left.+(1-\alpha) E_{R}\left[1_{B} \frac{C}{(1+r)^{T-t}} \mid \mathcal{F}_{t}\right]\right] \\
= & E_{Q}\left[\alpha 1_{B} X+(1-\alpha) 1_{B} Y\right] \\
= & E_{Q^{\prime}}\left[\alpha 1_{B} X+(1-\alpha) 1_{B} Y\right] .
\end{aligned}
$$

We find

$$
\mathcal{K}_{t} \ni E_{Q^{\prime}}\left[\frac{C}{(1+r)^{T-t}} \mid \mathcal{F}_{t}\right]=\alpha X+(1-\alpha) Y .
$$

Lemma 4.10 We have $\mathcal{C}_{0} \subseteq \Pi_{\mathbb{Z}}(C)$.

Proof Let $p \in \mathcal{C}_{0}$ and define $X_{0}:=p$. We can find recursively $X_{t+1} \in \mathcal{C}_{t+1}$ and $Q_{t} \in \mathfrak{Q}_{t}$ such that $X_{t}=E_{Q_{t}}\left[X_{t+1} /(1+r) \mid \mathcal{F}_{t}\right]$ for $t \in \mathbb{T} \backslash\{T\}$. Since $X_{T} \in \mathcal{C}_{T}=\{C\}$, we have $X_{T}=C$. Assume for a contradiction that there is an integer arbitrage for the model $\left(S^{0}, \ldots, S^{d}, X\right)$. By Lemma 2.4 , there is a one-period integer arbitrage $\left(\bar{\varphi}, \varphi^{d+1}\right)$, i.e., there is $t_{0} \in \mathbb{T}$ such that $\left(\varphi_{t}, \varphi_{t}^{d+1}\right)=0$ for any $t \in \mathbb{T} \backslash\left\{0, t_{0}\right\}$. Then 
there is a minimal set $B \in \mathcal{F}_{t_{0}-1} \backslash\{\emptyset\}$ such that $1_{B}\left(\bar{\varphi}, \varphi^{d+1}\right)$ is still an arbitrage. Define $\left(\eta, \eta^{d+1}\right):=\left(\varphi_{t_{0}}, \varphi_{t_{0}}^{d+1}\right)(\omega) \in \mathbb{Z}^{d+1}$ for some $\omega \in B$. Then

$$
Y:=1_{B}\left(\eta \Delta \hat{S}_{t_{0}}+\eta^{d+1}\left(\frac{X_{t_{0}}}{(1+r)^{t_{0}}}-\frac{X_{t_{0}-1}}{(1+r)^{t_{0}-1}}\right)\right) \geq 0
$$

and $P[Y>0]>0$. Since the model $\left(S^{0}, \ldots, S^{d}\right)$ satisfies NIA, we have $\eta^{d+1} \neq 0$ and can define $\xi^{j}:=\eta^{j} / \eta^{d+1}$. We get

$$
Y / \eta^{d+1}=1_{B}\left(\xi \Delta \hat{S}_{t_{0}}+\left(\frac{X_{t_{0}}}{(1+r)^{t_{0}}}-\frac{X_{t_{0}-1}}{(1+r)^{t_{0}-1}}\right)\right) .
$$

Thus $X_{t_{0}-1} \notin \mathcal{C}_{t_{0}-1}$, which is a contradiction.

It is not hard to see that actually $\mathcal{C}_{0}=\Pi_{\mathbb{Z}}(C)$, but we do not use this fact. $\operatorname{Re}$ call that $X^{*}$ is an adapted process such that $X_{0}^{*}=p, X_{t}^{*}=C$ and $\left(S^{0}, \ldots, S^{d}, X^{*}\right)$ satisfies NIA.

Lemma 4.11 Let $t \in \mathbb{T}$. Let $X_{t} \in \mathcal{K}_{t}$ and define $X_{t}^{\alpha}:=\alpha X_{t}+(1-\alpha) X_{t}^{*}$ for any $\alpha \in[0,1]$. Then there is a countable set $F \subseteq(0,1]$ such that $X_{t}^{\alpha} \in \mathcal{C}_{t}$ for any $\alpha \in[0,1] \backslash F$. In particular, $\mathcal{C}_{t}$ is dense in $\mathcal{K}_{t}$.

Proof Define

$$
I:=\{t \in \mathbb{T}: \text { the claim holds for } t\} .
$$

Obviously, $T \in I$. Let $t \in I \backslash\{0\}$. We show that $t-1 \in I$, which implies $I=\mathbb{T}$ and hence the claim. To this end, let $X_{t-1} \in \mathcal{K}_{t-1}$. Then there are $X_{t} \in \mathcal{K}_{t}$ and $Q \in \mathfrak{Q}_{t-1}$ such that $X_{t-1}=E_{Q}\left[X_{t} /(1+r) \mid \mathcal{F}_{t-1}\right]$. We define

$$
\begin{aligned}
X_{t-1}^{\alpha} & :=\alpha X_{t-1}+(1-\alpha) X_{t-1}^{*}, \\
X_{t}^{\alpha} & :=\alpha X_{t}+(1-\alpha) X_{t}^{*}
\end{aligned}
$$

for any $\alpha \in[0,1]$. There is $F_{t} \subseteq(0,1]$ countable such that $X_{t}^{\alpha} \in \mathcal{C}_{t}$ for any $\alpha \in[0,1] \backslash F_{t}$. For any $\alpha \in[0,1] \backslash F_{t}$, we find recursively $X_{s+1}^{\alpha} \in \mathcal{C}_{s+1}$ and $Q_{s}^{\alpha} \in \mathfrak{Q}_{s}$ such that $X_{s}^{\alpha}=E_{Q_{s}^{\alpha}}\left[X_{s+1}^{\alpha} /(1+r) \mid \mathcal{F}_{s}\right]$ for $s \geq t$.

We now show that $\left(S_{u}^{0}, \ldots, S_{u}^{d}, X_{u}^{\alpha}\right)_{u=t-1, \ldots, T}$ satisfies NIA for all but countably many choices for $\alpha \in[0,1]$. Since existence of an integer arbitrage implies existence of a one-period arbitrage and the market $\left(S_{u}^{0}, \ldots, S_{u}^{d}, X_{u}^{\alpha}\right)_{u=t, \ldots, T}$ does not allow arbitrage, we know that this arbitrage must be in the period from $t-1$ to $t$. Since $\mathcal{F}_{t-1}$ is generated by finitely many atoms, it is sufficient to condition on one of the atoms. Thus we may simply assume that $t=1$. We define

$$
F_{0}:=\left\{\alpha \in(0,1]: \alpha \in F_{1} \text { or } X_{0}^{\alpha} \notin \mathcal{C}_{0}\right\},
$$

and we show that $F_{0}$ is countable. To this end, we define the sets

$$
\begin{aligned}
\mathcal{D}_{1} & :=\left\{Y \in L^{0}\left(\left(\Omega, \mathcal{F}_{1}, P\right), \mathbb{R}\right): Y=\xi \Delta \hat{S}_{1} Q \text {-a.s., } \xi \in \mathbb{R}^{d}\right\}, \\
\mathcal{D}_{1}^{\mathbb{Q}}: & =\left\{Y \in L^{0}\left(\left(\Omega, \mathcal{F}_{1}, P\right), \mathbb{R}\right): Y=\xi \Delta \hat{S}_{1} Q \text {-a.s., } \xi \in \mathbb{Q}^{d}\right\},
\end{aligned}
$$

and $\Delta \hat{X}_{1}^{\alpha}:=\frac{X_{1}^{\alpha}}{1+r}-X_{0}^{\alpha}$ for $\alpha \in[0,1]$. 
Case 1: $\Delta \hat{X}_{1} \notin \mathcal{D}_{1}$ or $\Delta \hat{X}_{1}^{*} \notin \mathcal{D}_{1}$. We define $\tilde{F}:=\left\{\alpha \in[0,1]: \Delta X_{1}^{\alpha} \in \mathcal{D}_{1}\right\}$. Since $\mathcal{D}_{1}$ is a vector space, we find that $\bar{F}$ contains at most one element. We claim that $(0,1] \backslash\left(F_{1} \cup \bar{F}\right)$ does not contain any element of $F_{0}$. To show this, let $\alpha \in(0,1] \backslash\left(F_{1} \cup \bar{F}\right)$ and assume for a contradiction that there is an integer arbitrage in the first period. Hence there is $\xi \in \mathbb{Z}^{d+1}$ such that $\xi^{d+1} \neq 0$ and $\xi \Delta \hat{S}_{1}+\xi^{d+1} \Delta \hat{X}_{1}^{\alpha} \geq 0$. Since $Q$ is a martingale measure, we get $\xi \Delta \hat{S}_{1}+\xi^{d+1} \Delta \hat{X}_{1}^{\alpha}=0 Q$-a.s., and after solving for $\Delta \hat{X}_{1}^{\alpha}$, we find $\Delta \hat{X}_{1}^{\alpha} \in \mathcal{D}_{1}$, which is a contradiction.

Case 2: $\Delta \hat{X}_{1}, \Delta \hat{X}_{1}^{*} \in \mathcal{D}_{1}$ and there is a set with positive $Q$-measure on which $\Delta \hat{X}_{1} \neq \Delta \hat{X}_{1}^{*}$. Since $\mathcal{D}_{1}^{\mathbb{Q}}$ restricted to $\Omega \backslash A_{0}$ has countably many elements, we find that $\Delta \hat{X}_{1}^{\alpha} \in \mathcal{D}_{1}^{\mathbb{Q}}$ at most countably often. Denote by $\bar{F}$ the set of $\alpha \in[0,1]$ where $\Delta \hat{X}_{1}^{\alpha} \in \mathcal{D}_{1}^{\mathbb{Q}}$. We claim that $F_{0} \subseteq F_{1} \cup \bar{F}$. To show this, let $\alpha \in(0,1] \backslash\left(F_{1} \cup \bar{F}\right)$ and assume for a contradiction that there is an integer arbitrage in the first period. Then there is $\xi \in \mathbb{Z}^{d+1}$ such that $\xi^{d+1} \neq 0$ and $\xi \Delta \hat{S}_{1}+\xi^{d+1} \Delta \hat{X}_{1}^{\alpha} \geq 0$. Since $Q$ is a martingale measure, we get $\xi \Delta \hat{S}_{1}+\xi^{d+1} \Delta \hat{X}_{1}^{\alpha}=0 Q$-a.s., and after solving for $\Delta \hat{X}_{1}^{\alpha}$, we find $\Delta \hat{X}_{1}^{\alpha} \in \mathcal{D}_{1}^{\mathbb{Q}}$, which is a contradiction.

Case 3: $\Delta \hat{X}_{1}, \Delta \hat{X}_{1}^{*} \in \mathcal{D}_{1}$ and $\Delta \hat{X}_{1}=\Delta \hat{X}_{1}^{*} Q$-a.s. Then we have

$$
\begin{aligned}
X_{1} & =(1+r)\left(\Delta \hat{X}_{1}+X_{0}\right) \\
& =(1+r)\left(\Delta \hat{X}_{1}^{*}+X_{0}\right) \\
& =X_{1}^{*}+(1+r)\left(X_{0}-X_{0}^{*}\right) \quad Q \text {-a.s. }
\end{aligned}
$$

If $X_{0}=X_{0}^{*}$, then $X_{0}=X_{0}^{*} \in \mathcal{C}_{0}$. Thus we may assume that $X_{0} \neq X_{0}^{*}$. If $R[B] \in\{0,1\}$ for any $R \in \mathfrak{Q}_{\mathbb{Z}}^{\max }$ and any $B \in \mathcal{F}_{1}$, then Lemma 2.18 yields $\mathcal{F}_{1}=\mathcal{F}_{0}$ and hence $\Delta \hat{X}_{1}=0=\Delta \hat{X}_{1}^{*}$, which yields that $F_{0}=F_{1}$. Thus we may assume that there are $R \in \mathfrak{Q}_{\mathbb{Z}}^{\max }$ and $B \in \mathcal{F}_{1}$ with $R[B] \in(0,1)$. By Proposition 2.12 , we find that there is $B \in \mathcal{F}_{1}$ such that for any $R \in \mathfrak{Q}_{\mathbb{Z}}^{\max }=\mathfrak{Q}_{0}$, we have $R[B] \in(0,1)$. In particular, we have $Q[B] \in(0,1)$.

For $n \in \mathbb{N}$, we define

$$
\begin{aligned}
& Y_{1}^{n}:=X_{1} 1_{B^{\mathrm{c}}}+\left((1-1 / n) X_{1}+X_{1}^{*} / n\right) 1_{B}=X_{1}+1_{B}\left(X_{1}^{*}-X_{1}\right) / n, \\
& Y_{0}^{n}:=X_{0} Q\left[B^{\mathrm{c}}\right]+\left((1-1 / n) X_{0}+X_{0}^{*} / n\right) Q[B]=X_{0}+Q[B]\left(X_{0}^{*}-X_{0}\right) / n .
\end{aligned}
$$

Lemma 4.9 yields $Y_{1}^{n} \in \mathcal{K}_{1}$ for any $n \in \mathbb{N}$. The measure $Q_{n}[D]:=E_{Q}\left[Q_{n}^{\prime}\left[D \mid \mathcal{F}_{1}\right]\right]$, where $Q_{n}^{\prime} \in \mathfrak{Q}_{1}$ is such that $Y_{1}^{n}=E_{Q_{n}^{\prime}}\left[\frac{C}{(1+r)^{T-1}} \mid \mathcal{F}_{1}\right]$, satisfies

$$
E_{Q_{n}}\left[\frac{Y_{1}^{n}}{1+r}\right]=E_{Q}\left[\frac{Y_{1}^{n}}{1+r}\right]=Y_{0}^{n},
$$

where the last equality follows from the definition of $Q$ and (4.1). Thus $Y_{0}^{n} \in \mathcal{K}_{0}$ for any $n \in \mathbb{N}$. Observe that

$$
\Delta \hat{Y}_{1}^{n}:=\frac{Y_{1}^{n}}{1+r}-Y_{0}^{n}=\Delta \hat{X}_{1}+\frac{1}{n}\left(1_{B} \frac{X_{1}^{*}-X_{1}}{1+r}-Q[B]\left(X_{0}^{*}-X_{0}\right)\right) .
$$


We find that $\Delta \hat{Y}_{1}^{n} \neq \Delta \hat{X}_{1}^{*}$ with positive $Q$-probability. By appealing to Case 1 (resp. Case 2), we find $F^{n} \subseteq[0,1]$ countable such that $\alpha Y_{0}^{n}+(1-\alpha) X_{0}^{*} \in \mathcal{C}_{0}$ for any $\alpha \in[0,1] \backslash F^{n}$. Define the countable set

$$
\bar{F}:=\left\{\alpha(1-Q[B] / n): n \in \mathbb{N}, \alpha \in F^{n}\right\} .
$$

We claim that $F_{0} \backslash\{1\} \subseteq F_{1} \cup \bar{F}$. To show this, let $\alpha \in(0,1) \backslash\left(F_{1} \cup \bar{F}\right)$. Choose $n \in \mathbb{N}$ such that $n>Q[B] /(1-\alpha)$. Then there is $\alpha^{\prime} \in[0,1]$ such that $\alpha=\alpha^{\prime}(1-Q[B] / n)$. We find $\alpha^{\prime} \notin F^{n}$, because $\alpha \notin \bar{F}$. Thus we have

$$
\begin{aligned}
\mathcal{C}_{0} \ni \alpha^{\prime} Y_{0}^{n}+\left(1-\alpha^{\prime}\right) X_{0}^{*} & =X_{0}^{*}+\frac{\alpha}{1-Q[B] / n}\left(Y_{0}^{n}-X_{0}^{*}\right) \\
& =X_{0}^{*}+\frac{\alpha}{1-Q[B] / n}\left(X_{0}-X_{0}^{*}\right)(1-Q[B] / n) \\
& =X_{0}^{*}+\alpha\left(X_{0}-X_{0}^{*}\right) \\
& =X_{0}^{\alpha} .
\end{aligned}
$$

Consequently, $\alpha \notin F_{0}$, and we have shown that $F_{0}$ is countable.

\section{Integer superhedging}

In this section, we discuss some properties of the integer superhedging price $\sigma_{\mathbb{Z}}(C)$ of a claim, as defined in (3.1). First, we give a simple example where it does not agree with the classical superhedging price sup $\Pi(C)$.

Example 5.1 In this example, the gap between sup $\Pi(C)$ and the cheapest integer superhedging price $\sigma_{\mathbb{Z}}(C)$ has size $a$, for an arbitrary number $a>0$. On the probability space $\Omega=\left\{\omega_{1}, \omega_{2}\right\}$, consider the one-dimensional model

$$
S_{0}^{1}=3 a, \quad S_{1}^{1}\left(\omega_{1}\right)=a, \quad S_{1}^{1}\left(\omega_{2}\right)=5 a
$$

with $r=0$. The unique equivalent martingale measure is $\left(\delta_{\omega_{1}}+\delta_{\omega_{2}}\right) / 2$, and so the unique arbitrage-free price of the claim

$$
C\left(\omega_{1}\right)=0, \quad C\left(\omega_{2}\right)=2 a
$$

is given by $\Pi(C)=\{a\}$. By Theorem 3.6, we have $\Pi_{\mathbb{Z}}(C)=\Pi(C)=\{a\}$. The integer superhedging price is found by computing

$$
\sigma_{\mathbb{Z}}(C)=\inf _{\varphi \in \mathbb{Z}} \max _{\omega \in \Omega}\left(C(\omega)-\varphi \Delta S_{1}^{1}(\omega)\right)=\min _{\varphi \in \mathbb{Z}} \max (2 a \varphi, 2 a-2 a \varphi)=2 a .
$$

We obtain that the interval of prices of integer superhedges is $[2 a, \infty)$. For real $\varphi$, the minimum in (5.1) is attained at $\varphi=\frac{1}{2}$, yielding the classical superhedging price $a=\sup \Pi(C)$. 
As soon as a model is fixed, the gap considered in the preceding example can be bounded for all claims. In Example 5.1, we have equality in (5.2) below. On $\mathbb{R}^{n}$, we always use the Euclidean norm $\|\cdot\|=\|\cdot\|_{2}$.

Proposition 5.2 Under Assumption 2.1 and $N A$, we have for any claim $C$ that

$$
\sigma_{\mathbb{Z}}(C)-\sup \Pi(C) \leq \frac{1}{2} \sqrt{d} \max _{\omega \in \Omega} \sum_{k=1}^{T}\left\|\Delta \hat{S}_{k}(\omega)\right\| .
$$

Proof Let $\bar{\psi} \in \mathcal{R}$ be a cheapest classical superhedge. By Theorem 3.2, it satisfies $V_{0}(\bar{\psi})=\sup \Pi(C)$. By rounding the risky positions of $\bar{\psi}$ to the closest integers (with any convention for half-integers), we get a strategy $\left(\psi^{0},\lfloor\psi\rceil\right) \in \mathcal{Z}$. Clearly,

$$
\left\|\psi_{t}-\lfloor\psi\rceil_{t}\right\| \leq\left\|\left(\frac{1}{2}, \ldots, \frac{1}{2}\right)\right\|=\frac{1}{2} \sqrt{d}, \quad t \in \mathbb{T} .
$$

Define $\hat{C}=C /(1+r)^{T}$ and let $\bar{\varphi} \in \mathcal{Z}$. Since $\hat{V}_{T}(\bar{\varphi})=V_{0}(\bar{\varphi})+\sum_{k=1}^{T} \varphi_{k} \Delta \hat{S}_{k}$, we get the necessary condition

$$
V_{0}(\bar{\varphi})=\max _{\omega \in \Omega}\left(\hat{C}(\omega)-\sum_{k=1}^{T} \varphi_{k}(\omega) \Delta \hat{S}_{k}(\omega)\right),
$$

if $\bar{\varphi}$ were a cheapest integer superhedge. It follows that

$$
\begin{aligned}
\sigma_{\mathbb{Z}}(C)= & \inf _{\substack{\varphi \text { predictable, } \\
\mathbb{Z}^{d} \text {-valued }}} \max _{\omega \in \Omega}\left(\hat{C}(\omega)-\sum_{k=1}^{T} \varphi_{k}(\omega) \Delta \hat{S}_{k}(\omega)\right) \\
\leq & \max _{\omega \in \Omega}\left(\hat{C}(\omega)-\sum_{k=1}^{T}\lfloor\psi\rceil_{k}(\omega) \Delta \hat{S}_{k}(\omega)\right) \\
\leq & \max _{\omega \in \Omega}\left(\hat{C}(\omega)-\sum_{k=1}^{T} \psi_{k}(\omega) \Delta \hat{S}_{k}(\omega)\right) \\
& +\max _{\omega \in \Omega} \sum_{k=1}^{T}\left(\psi_{k}(\omega)-\lfloor\psi\rceil_{k}(\omega)\right) \Delta \hat{S}_{k}(\omega) \\
\leq & \sup \Pi(C)+\max _{\omega \in \Omega} \sum_{k=1}^{T}\left\|\psi_{k}(\omega)-\lfloor\psi\rceil_{k}(\omega)\right\|\left\|\Delta \hat{S}_{k}(\omega)\right\| \\
\leq & \sup \Pi(C)+\frac{1}{2} \sqrt{d} \max _{\omega \in \Omega} \sum_{k=1}^{T}\left\|\Delta \hat{S}_{k}(\omega)\right\| .
\end{aligned}
$$

The following example shows that contrary to the case of classical superhedging, there need not exist a cheapest integer superhedge. 
Example 5.3 Let $\Omega=\left\{\omega_{1}, \omega_{2}\right\}, r=0, T=1$ and $d=2$. We choose the model with $S_{0}:=(2,2)$ and

$$
S_{1}\left(\omega_{j}\right)= \begin{cases}(3,2-\sqrt{2}), & j=1, \\ (1,2+\sqrt{2}), & j=2 .\end{cases}
$$

This model satisfies NA, and it is complete in the classical sense. Indeed, the only martingale measure is given by $Q\left[\left\{\omega_{j}\right\}\right]=1 / 2$ for $j=1,2$. Consider the claim

$$
C\left(\omega_{1}\right)=1-\frac{1}{2} \sqrt{2}, \quad C\left(\omega_{2}\right)=1+\frac{1}{2} \sqrt{2},
$$

whose set of (integer-) arbitrage-free prices is the singleton

$$
\Pi_{\mathbb{Z}}(C)=\Pi(C)=\{1\} .
$$

Then there is no minimiser for the superhedging problem (see (5.3))

$$
\inf _{\varphi \in \mathbb{Z}^{2}} \max _{i=1,2}\left(C\left(\omega_{i}\right)-\varphi \Delta S_{1}\left(\omega_{i}\right)\right)=\inf _{\varphi \in \mathbb{Z}^{2}} f(\varphi)
$$

with

$$
f(\varphi):=\max \left(1-\frac{1}{2} \sqrt{2}-\varphi^{1}+\sqrt{2} \varphi^{2}, 1+\frac{1}{2} \sqrt{2}+\varphi^{1}-\sqrt{2} \varphi^{2}\right) .
$$

Indeed, for $\varphi \in \mathbb{R}^{2}$, the set of minimisers would be

$$
\left\{\left(x, \frac{1}{2}+x / \sqrt{2}\right): x \in \mathbb{R}\right\}
$$

yielding $\inf _{\varphi \in \mathbb{R}^{2}} f(\varphi)=1$. Obviously, this set contains no integer strategies. By Kronecker's approximation theorem (Theorem A.2), the sequence $\left(\frac{1}{2}+m / \sqrt{2}\right) \bmod 1$, $m \in \mathbb{N}$, is dense in $[0,1]$. Thus there is a sequence $\left(m_{k}\right) \subseteq \mathbb{N}$ such that

$$
0 \leq\left(\frac{1}{2}+m_{k} / \sqrt{2}\right) \bmod 1 \leq \frac{1}{k}, \quad k \in \mathbb{N} .
$$

Define

$$
\varphi^{(k)}:=\left(m_{k},\left\lfloor\frac{1}{2}+m_{k} / \sqrt{2}\right\rfloor\right) \in \mathbb{Z}^{2}, \quad k \in \mathbb{N} .
$$

Since $f$ is Lipschitz-continuous (with constant $L$, say), we have

$$
\begin{aligned}
\left|f\left(\varphi^{(k)}\right)-1\right| & =\left|f\left(\varphi^{(k)}\right)-f\left(m_{k}, \frac{1}{2}+m_{k} / \sqrt{2}\right)\right| \\
& \leq L\left\|\left(0,\left(\frac{1}{2}+m_{k} / \sqrt{2}\right) \bmod 1\right)\right\| \longrightarrow 0, \quad k \rightarrow \infty .
\end{aligned}
$$

Thus the infimum of the prices of integer superhedges is $\sigma_{\mathbb{Z}}(C)=1$, but there is no cheapest integer superhedge. 
Financial institutions usually hedge large portfolios of identical (or at least similar) options. The following theorem shows that when superhedging $N$ copies of $C$, the integer superhedging price per claim converges to the classical superhedging price, i.e., $\lim _{N \rightarrow \infty} N^{-1} \sigma_{\mathbb{Z}}(N C)=\sup \Pi(C)$. The second part of Theorem 5.4 gives an estimate on superhedging $C$ with rational strategies with controlled denominators.

Theorem 5.4 Suppose that Assumption 2.1 and NA hold, and let $C$ be a claim. Then:

(i)

$$
\frac{\sigma_{\mathbb{Z}}(N C)}{N}=\sup \Pi(C)+O\left(\frac{1}{N}\right), \quad N \rightarrow \infty .
$$

(ii) There is a sequence of rational strategies $\bar{\psi}^{(N)} \in \mathcal{Q}$ such that all denominators occurring in $\bar{\psi}^{(N)}$ have absolute value at most $N$,

$$
V_{0}\left(\bar{\psi}^{(N)}\right)=\sup \Pi(C)+O\left(N^{-1 /(n d(T+1))} \log N\right),
$$

and $\bar{\psi}^{(N)}$ is a superhedging strategy for $C$.

Proof (i) Assumption 2.1 implies that the classical superhedging price

$$
\sup \Pi(C)=\sup \left\{E_{Q}\left[C /(1+r)^{T}\right]: Q \in \mathfrak{Q}\right\}
$$

is finite. It is clear that

$$
N^{-1} \sigma_{\mathbb{Z}}(N C) \geq N^{-1} \sup \Pi(N C)=\sup \Pi(C)
$$

for all $N$. For the converse estimate, let $\bar{\varphi}$ be a classical superhedging strategy for $C$ with price sup $\Pi(C)$ (see Theorem 3.2). Define

$$
\eta_{t}^{(N), j}(\omega):=\left\lfloor N \varphi_{t}^{j}(\omega)\right\rfloor=N \varphi_{t}^{j}(\omega)+O(1), \quad \omega \in \Omega, t \in \mathbb{T}, 1 \leq j \leq d, N \in \mathbb{N} .
$$

We choose an arbitrary map $f: \mathbb{N} \rightarrow \mathbb{R}$ satisfying $\lim _{N \rightarrow \infty} f(N)=\infty$ and put

$$
\eta_{1}^{(N), 0}:=N \varphi_{1}^{0}+f(N), \quad N \in \mathbb{N} .
$$

Then we define $\eta_{t}^{(N), 0}$ for $t=2, \ldots, T$ recursively to obtain a self-financing integer strategy $\bar{\eta}^{(N)}$ for each $N$. By the definition of $\eta^{(N)}$ and since $\bar{\varphi}$ is a superhedging strategy, we have

$$
\begin{aligned}
\frac{\hat{V}_{T}\left(\bar{\eta}^{(N)}\right)}{N} & =\frac{V_{0}\left(\bar{\eta}^{(N)}\right)}{N}+\sum_{k=1}^{T} \frac{\eta_{k}^{(N)}}{N} \Delta \hat{S}_{k} \\
& =\varphi_{1}^{0}+\frac{f(N)}{N}+\frac{\eta_{1}^{(N)} S_{0}}{N}+\sum_{k=1}^{T} \varphi_{k} \Delta \hat{S}_{k}+O\left(\frac{1}{N}\right) \\
& =\frac{f(N)}{N}+V_{0}(\bar{\varphi})+\sum_{k=1}^{T} \varphi_{k} \Delta \hat{S}_{k}+O\left(\frac{1}{N}\right) \\
& \geq \frac{C}{(1+r)^{T}}+\frac{f(N)}{N}+O\left(\frac{1}{N}\right) \geq \frac{C}{(1+r)^{T}}
\end{aligned}
$$


for large $N$. This shows that $\bar{\eta}^{(N)}$ is an integer superhedging strategy for $N C$ for large $N$, and hence

$$
\begin{aligned}
\sigma_{\mathbb{Z}}(N C) & \leq V_{0}\left(\bar{\eta}^{(N)}\right) \\
& =N V_{0}(\bar{\varphi})+O(f(N)) \\
& =N \sup \Pi(C)+O(f(N)), \quad N \rightarrow \infty .
\end{aligned}
$$

It is easy to see that a quantity that is $O(f(N))$ for any $f$ tending to infinity is $O(1)$. Since $f$ was arbitrary, the statement follows.

(ii) Again, let $\bar{\varphi}$ be a classical superhedging strategy for $C$ with price sup $\Pi(C)$. By Dirichlet's approximation theorem (Theorem A.1), there are $1 \leq q(N) \leq N$ and $p(N, t, j, \ell) \in \mathbb{Z}$ such that for $1 \leq \ell \leq n, t \in \mathbb{T}, 1 \leq j \leq d, N \in \mathbb{N}$, we have

$$
\left|\varphi_{t}^{j}\left(\omega_{\ell}\right) q(N)-p(N, t, j, \ell)\right|<N^{-1 /(n d(1+T))} .
$$

We define

$$
\psi_{t}^{(N), j}\left(\omega_{\ell}\right):=\frac{p(N, t, j, \ell)}{q(N)}, \quad 1 \leq \ell \leq n, t \in \mathbb{T}, 1 \leq j \leq d, N \in \mathbb{N},
$$

which yields

$$
\left|\varphi_{t}^{j}\left(\omega_{\ell}\right)-\psi_{t}^{(N), j}\left(\omega_{\ell}\right)\right|<N^{-1 /(n d(1+T))}, \quad 1 \leq \ell \leq n, t \in \mathbb{T}, 1 \leq j \leq d, N \in \mathbb{N} .
$$

After fixing the initial bank account position

$$
\psi_{1}^{(N), 0}:=\varphi_{1}^{0}+N^{-1 /(n d(1+T))} \log N, \quad N \in \mathbb{N},
$$

a strategy $\bar{\psi}^{(N)} \in \mathcal{Q}$ is defined for each $N$. By definition,

$$
\begin{aligned}
V_{0}\left(\bar{\psi}^{(N)}\right) & =\varphi_{1}^{0}+N^{-1 /(n d(1+T))} \log N+\psi_{1}^{(N)} S_{0} \\
& =\varphi_{1}^{0}+N^{-1 /(n d(1+T))} \log N+\varphi_{1} S_{0}+O\left(N^{-1 /(n d(1+T))}\right) \\
& =\sup \Pi(C)+O\left(N^{-1 /(n d(1+T))} \log N\right), \quad N \rightarrow \infty .
\end{aligned}
$$

It remains to show that $\bar{\psi}^{(N)}$ is a superhedge for $C$ for large $N$. This follows from

$$
\begin{aligned}
\hat{V}_{T}\left(\bar{\psi}^{(N)}\right) & =V_{0}\left(\bar{\psi}^{(N)}\right)+\sum_{k=1}^{T} \psi_{k}^{(N)} \Delta \hat{S}_{k} \\
& =V_{0}(\bar{\varphi})+N^{-1 /(n d(1+T))} \log N+\sum_{k=1}^{T} \varphi_{k} \Delta \hat{S}_{k}+O\left(N^{-1 /(n d(1+T))}\right) \\
& \geq \frac{C}{(1+r)^{T}}+N^{-1 /(n d(1+T))} \log N+O\left(N^{-1 /(n d(1+T))}\right) \\
& \geq \frac{C}{(1+r)^{T}} \quad \text { for } N \text { large. }
\end{aligned}
$$


For those finitely many $N$ where the last inequality does not hold, we can simply add a sufficient amount of initial capital to obtain a superhedge; this does not change the convergence rate.

From the proof of (ii), it is clear that $\log N$ can be replaced by an arbitrary function tending to infinity.

\section{Variance-optimal hedging in one period}

We consider a one-period model satisfying Assumption 2.1 and NA. Moreover, we suppose that $d \leq n$. Our goal is to approximately hedge a given (non-replicable) claim $C$. For tractability, the error is measured by the norm of $L^{2}\left(P^{*}\right)$, where $P^{*}$ is a fixed EMM; we denote this norm by $\|\cdot\|$ throughout this section. In the classical case, this leads to the optimisation problem

$$
\inf _{\varphi \in \mathbb{R}^{d}} \inf _{V_{0} \in \mathbb{R}}\left\|C /(1+r)-V_{0}-\varphi \Delta S_{1}\right\|=\inf _{\varphi \in \mathbb{R}^{d}}\left\|\tilde{C}-\varphi \Delta S_{1}\right\|,
$$

where $\tilde{C}:=\left(C-E^{*}[C]\right) /(1+r)$. Note that $\inf _{V_{0} \in \mathbb{R}}$ is attained at

$$
V_{0}=E^{*}\left[C /(1+r)-\varphi \Delta S_{1}\right]=E^{*}[C] /(1+r) .
$$

The problem (6.1) is then solved by projecting the claim $\tilde{C}$ orthogonally onto the space $\left\{\varphi \Delta S_{1}: \varphi \in \mathbb{R}^{d}\right\}$, which is closed by Theorem 6.4.2 in [10]. For more details on variance-optimal hedging (in particular, on the multi-period problem), we refer to Chap. 10 of [13] and the references given there.

Now we proceed to our setup and restrict $\varphi$ to $\mathbb{Z}^{d}$. The minimisation with respect to $V_{0}$ is done as in (6.1), and we thus have to compute

$$
\inf _{\varphi \in \mathbb{Z}^{d}}\left\|\tilde{C}-\varphi \Delta S_{1}\right\|
$$

We have

$$
\begin{aligned}
\left\|\tilde{C}-\varphi \Delta S_{1}\right\|^{2} & =\sum_{\ell=1}^{n} P^{*}\left[\left\{\omega_{\ell}\right\}\right]\left(\tilde{C}\left(\omega_{\ell}\right)-\varphi \Delta S_{1}\left(\omega_{\ell}\right)\right)^{2} \\
& =\sum_{\ell=1}^{n}\left(\tilde{C}\left(\omega_{\ell}\right) P^{*}\left[\left\{\omega_{\ell}\right\}\right]^{1 / 2}-\varphi \Delta S_{1}\left(\omega_{\ell}\right) P^{*}\left[\left\{\omega_{\ell}\right\}\right]^{1 / 2}\right)^{2} .
\end{aligned}
$$

The problem (6.2) thus amounts to computing the element of the lattice

$$
\left\{\varphi^{1}\left(\begin{array}{c}
\Delta S_{1}^{1}\left(\omega_{1}\right) P^{*}\left[\left\{\omega_{1}\right\}\right]^{1 / 2} \\
\vdots \\
\Delta S_{1}^{1}\left(\omega_{n}\right) P^{*}\left[\left\{\omega_{n}\right\}\right]^{1 / 2}
\end{array}\right)+\cdots+\varphi^{d}\left(\begin{array}{c}
\Delta S_{1}^{d}\left(\omega_{1}\right) P^{*}\left[\left\{\omega_{1}\right\}\right]^{1 / 2} \\
\vdots \\
\Delta S_{1}^{d}\left(\omega_{n}\right) P^{*}\left[\left\{\omega_{n}\right\}\right]^{1 / 2}
\end{array}\right): \varphi \in \mathbb{Z}^{d}\right\}
$$


Table 1 Model parameters, risk-neutral measure and a claim

\begin{tabular}{lllll}
\hline$i$ & 1 & 2 & 3 & 4 \\
\hline$P^{*}\left[\left\{\omega_{i}\right\}\right]$ & 0.37 & 0.18 & 0.4 & 0.05 \\
$\Delta S^{1}\left(\omega_{i}\right)$ & -9 & -9 & 0 & 99 \\
$\Delta S^{2}\left(\omega_{i}\right)$ & 10 & 1 & 4 & -109.6 \\
$C\left(\omega_{i}\right)$ & 0 & 7 & 1 & 8 \\
\hline
\end{tabular}

closest to the vector

$$
\left(\begin{array}{c}
\tilde{C}\left(\omega_{1}\right) P^{*}\left[\left\{\omega_{1}\right\}\right]^{1 / 2} \\
\vdots \\
\tilde{C}\left(\omega_{n}\right) P^{*}\left[\left\{\omega_{n}\right\}\right]^{1 / 2}
\end{array}\right) \in \mathbb{R}^{n}
$$

with respect to the Euclidean norm. This is an instance of the closest vector problem (CVP), a well-known computational problem with applications in cryptography, communications theory and other fields. The survey paper [15] offers an accessible introduction to this subject with many references. By the Pythagorean theorem, the closest lattice point is the lattice point closest to the projection of (6.4) to the subspace generated by the lattice. A cheap method to compute a (hopefully) close lattice point consists of rounding the coefficients of this projected point to the closest integers. It is well known, though, that the resulting point may be far from optimal. This happens in the following example.

We consider a toy example with $d=2,|\Omega|=4$ and $r=0$, specified in Table 1 . The numbers are not calibrated to any market data, but are chosen to illustrate the point that a naive approach at integer approximate hedging (as mentioned above) can lead to significant errors. A detailed investigation of integer variance-optimal hedging over several periods in realistic models is left to future work.

We wish to approximately hedge $N$ copies of the claim, i.e., the claim $N C$, for $N \in \mathbb{N}$. First, we compute the classical variance-optimal hedge $\varphi^{(N)}=N \varphi^{(1)} \in \mathbb{R}^{2}$ by projection (see (6.1)). The relative $L^{2}\left(P^{*}\right)$-error

$$
\frac{\left\|N \tilde{C}-\varphi^{(N)} \Delta S_{1}\right\|}{\|N \tilde{C}\|}=\frac{\left\|\tilde{C}-\varphi^{(1)} \Delta S_{1}\right\|}{\|\tilde{C}\|},
$$

which of course does not depend on $N$, is displayed in the second lines of Tables 2 and 3. Then, we solve the integer variance-optimal hedging problem (6.2) exactly, using the algorithm CLOSESTPOINT described in [1], which is based on the SchnorrEuchner algorithm [20]. CVP is known as a computationally hard problem, with the fastest known algorithms having exponential complexity in the dimension. Since our dimension is only $|\Omega|=4$, this was not an issue in this toy example. In more sophisticated examples, a preprocessing using the LLL-algorithm [18] might facilitate the task of computing a closest vector. The fourth lines of Tables 2 and 3 show the relative $L^{2}\left(P^{*}\right)$-error

$$
\frac{\left\|N \tilde{C}-\varphi_{\mathrm{CVP}}^{(N)} \Delta S_{1}\right\|}{\|N \tilde{C}\|} .
$$


Table 2 Errors and trading strategies for variance-optimal hedging

\begin{tabular}{lllll}
\hline$N$ & 1 & 5 & 10 & 20 \\
\hline Classical: rMSE & 0.405 & 0.405 & 0.405 & 0.405 \\
Classical: strategy & $(-0.49,-0.51)$ & $(-2.49,-2.55)$ & $(-4.98,-5.10)$ & $(-9.97,-10.21)$ \\
CVP: rMSE & 0.901 & 0.431 & 0.419 & 0.416 \\
CVP: strategy & $(-1,-1)$ & $(-3,-3)$ & $(-5,-5)$ & $(-11,-11)$ \\
Rounding: rMSE & 8.352 & 1.636 & 0.419 & 0.419 \\
Rounding: strategy & $(0,-1)$ & $(-2,-3)$ & $(-5,-5)$ & $(-10,-10)$ \\
\hline
\end{tabular}

Table 3 Errors and trading strategies for variance-optimal hedging

\begin{tabular}{llll}
\hline$N$ & 30 & 40 & 50 \\
\hline Classical: rMSE & 0.405 & 0.405 & 0.405 \\
Classical: strategy & $(-14.96,-15.31)$ & $(-19.94,-20.42)$ & $(-24.93,-25.53)$ \\
CVP: rMSE & 0.415 & 0.415 & 0.410 \\
CVP: strategy & $(-16,-16)$ & $(-21,-21)$ & $(-24,-25)$ \\
Rounding: rMSE & 0.419 & 0.419 & 0.412 \\
Rounding: strategy & $(-15,-15)$ & $(-20,-20)$ & $(-25,-26)$ \\
\hline
\end{tabular}

Finally, we used a poor man's approach at solving (6.2) approximately, by simply rounding the positions of the classical hedge $\varphi^{(N)}$ to the closest integers. From Table 2, we see that this works fine for large $N$, but gives significantly worse results than solving CVP for small $N$. To conclude, we mention that computing the so-called covering radius [15] of the lattice (6.3) yields an upper bound for the hedging error for any claim.

Acknowledgements We thank the anonymous referees and the editors for very useful comments.

Open Access This article is distributed under the terms of the Creative Commons Attribution 4.0 International License (http://creativecommons.org/licenses/by/4.0/), which permits unrestricted use, distribution, and reproduction in any medium, provided you give appropriate credit to the original author(s) and the source, provide a link to the Creative Commons license, and indicate if changes were made.

\section{Appendix: Tools from number theory}

In this appendix, we collect the classical number-theoretic theorems we have used in this paper. The theorems of Dirichlet and Kronecker are fundamental results in Diophantine approximation (i.e., the approximation of real numbers by rational numbers).

Theorem A.1 (Dirichlet's approximation theorem; Theorem II.1B in [19]) Given $\alpha_{1}, \ldots, \alpha_{n} \in \mathbb{R}$ and an integer $N>1$, there are $q, x_{1}, \ldots, x_{n} \in \mathbb{Z}$ with $1 \leq q \leq N$ and

$$
\left|\alpha_{i} q-x_{i}\right|<N^{-1 / n}, \quad 1 \leq i \leq n
$$


Theorem A.2 (Kronecker's approximation theorem; Theorem 7.7 in [2]) If $\theta \in \mathbb{R}$ is an irrational number, then the sequence $(n \theta \bmod 1)_{n \in \mathbb{N}}$ is dense in $[0,1]$.

We also mention here the following classical theorem [14, Chap. 2, Theorem 1].

Theorem A.3 (Minkowski) Let $\mathcal{K} \subseteq \mathbb{R}^{d}$ be closed, convex, zero-symmetric and bounded. If the volume of $\mathcal{K}$ satisfies $\operatorname{vol}(\mathcal{K}) \geq 2^{d}$, then $\mathcal{K}$ contains a non-zero point with integer coordinates.

We did not use Theorem A.3 in this paper, but hint at a possible application. Consider the one-period portfolio optimisation problem with maximum loss constraint, where $c>0$ and $U$ is some utility function, i.e.,

$$
E\left[U\left(\varphi \Delta S_{1}\right)\right] \rightarrow \max ! \quad \text { over } \varphi \in \mathbb{Z}^{d} \text { subject to } \varphi \Delta S_{1} \geq-c .
$$

Then Theorem A.3 easily yields a sufficient criterion to ensure that the admissibility set contains a non-zero portfolio. Refinements of Theorem A.3 give several linearly independent portfolios.

\section{References}

1. Agrell, E., Eriksson, T., Vardy, A., Zeger, K.: Closest point search in lattices. IEEE Trans. Inf. Theory 48, 2201-2214 (2002)

2. Apostol, T.M.: Modular Functions and Dirichlet Series in Number Theory, 2nd edn. Graduate Texts in Mathematics, vol. 41. Springer, New York (1990)

3. Baumann, P., Trautmann, N.: Portfolio-optimization models for small investors. Math. Methods Oper. Res. 77, 345-356 (2013)

4. Beck, M., Robins, S.: Computing the Continuous Discretely, 2nd edn. Undergraduate Texts in Mathematics. Springer, New York (2015)

5. Bender, C., Kohlmann, M.: Optimal superhedging under non-convex constraints-a BSDE approach. Int. J. Theor. Appl. Finance 11, 363-380 (2008)

6. Bienstock, D.: Computational study of a family of mixed-integer quadratic programming problems. Math. Program. 74(2, Ser. A), 121-140 (1996)

7. Bonami, P., Lejeune, M.A.: An exact solution approach for portfolio optimization problems under stochastic and integer constraints. Oper. Res. 57, 650-670 (2009)

8. Carassus, L., Pham, H., Touzi, N.: No arbitrage in discrete time under portfolio constraints. Math. Finance 11, 315-329 (2001)

9. Dalang, R.C., Morton, A., Willinger, W.: Equivalent martingale measures and no-arbitrage in stochastic securities market models. Stoch. Stoch. Rep. 29, 185-201 (1990)

10. Delbaen, F., Schachermayer, W.: The Mathematics of Arbitrage. Springer, Berlin (2006)

11. Deng, X., Li, Z., Wang, S.: Computational complexity of arbitrage in frictional security market. Int. J. Found. Comput. Sci. 13, 681-684 (2002)

12. Föllmer, H., Kramkov, D.: Optional decompositions under constraints. Probab. Theory Relat. Fields 109, 1-25 (1997)

13. Föllmer, H., Schied, A.: Stochastic Finance, 4th edn. de Gruyter, Berlin (2016)

14. Gruber, P.M., Lekkerkerker, C.G.: Geometry of Numbers, 2nd edn. North-Holland, Amsterdam (1987)

15. Hanrot, G., Pujol, X., Stehlé, D.: Algorithms for the shortest and closest lattice vector problems. In: Chee, Y.M., et al. (eds.) Coding and Cryptology. Lecture Notes in Comput. Sci., vol. 6639, pp. 159190. Springer, Heidelberg (2011)

16. Harrison, J.M., Kreps, D.M.: Martingales and arbitrage in multiperiod securities markets. J. Econ. Theory 20, 381-408 (1979) 
17. Kabanov, Y., Stricker, C.: A teachers' note on no-arbitrage criteria. In: Azéma, J., et al. (eds.) Séminaire de Probabilités XXXV. Lecture Notes in Math., vol. 1755, pp. 149-152. Springer, Berlin (2001)

18. Nguyen, P.Q., Vallée, B. (eds.): The LLL Algorithm. Information Security and Cryptography. Springer, Berlin (2010)

19. Schmidt, W.M.: Diophantine Approximations and Diophantine Equations. Lecture Notes in Mathematics, vol. 1467. Springer, Berlin (1991)

20. Schnorr, C.-P., Euchner, M.: Lattice basis reduction: improved practical algorithms and solving subset sum problems. Math. Program. 66(2, Ser. A), 181-199 (1994)

21. Stanley, R.P.: Enumerative Combinatorics, vol. 1, 2nd edn. Cambridge Studies in Advanced Mathematics, vol. 49. Cambridge University Press, Cambridge (2012) 